\title{
Analyticity of Correlation Functions for the Two-Dimensional Ising Model
}

\section{Huzihiro Araki}

Research Institute for Mathematical Sciences, Kyoto University, Kyoto, 606 Japan

\begin{abstract}
Analyticity of correlation functions for the two-dimensional Ising model as a function of the inverse temperature except for the singularity at the critical temperature is proved. A crucial step is the establishment of the correspondence between extremal equilibrium states of the model and pure ground states of a one-dimensional spin system below the critical temperature $T_{c}$. An exact decay rate of the clustering property along axes is also determined for all $T \neq T_{c}$.
\end{abstract}

\section{Main Results}

We consider the two-dimensional Ising model with the Hamiltonian

$$
H(\xi)=-\sum_{i, j}\left(J_{1} \xi_{i j} \xi_{i+1, j}+J_{2} \xi_{i j} \xi_{i, j+1}\right) \text {, }
$$

where $\xi_{i j}= \pm 1,(i, j) \in \mathbb{Z}^{2}$, and $J$ 's are real constants. We are interested in the thermodynamic limit $(L, M \rightarrow \infty)$

$$
\begin{gathered}
\psi_{\beta}(F)=\lim \langle F\rangle_{L M}, \quad\langle F\rangle_{L M}=Z_{L M}^{-1} \sum_{\xi} F(\xi) e^{-\beta H^{L M}(\xi)}, \\
Z_{L M}=\sum_{\xi} \exp -\beta H^{L M}(\xi)
\end{gathered}
$$

in which $H^{L M}$ denotes (1.1) with the sum over $\xi_{k l}$ with $(k, l) \in[-L, L] \times[-M, M]$ and we consider an arbitrary polynomial $F(\xi)$ of a finite number of $\xi$ 's, which we call a strictly local observable.

There is a critical inverse temperature $\beta_{c}$ such that $\psi_{\beta}$ is the unique equilibrium state for $|\beta|<\beta_{c}$ while there exist two extremal equilibrium states $\psi_{\beta \pm}$ with

$$
\psi_{\beta}=\left(\psi_{\beta+}+\psi_{\beta-}\right) / 2
$$

for $|\beta|>\beta_{c}[1,12]$. Our main result is as follows: 
Theorem 1. For any strictly local $F, \psi_{\beta}(F)$ for $|\beta| \neq \beta_{c}$ and $\psi_{\beta \pm}(F)$ for $|\beta|>\beta_{c}$ are real analytic in $\beta, J_{1}$, and $J_{2}$.

Let $\tau_{(l, m)}$ be the lattice translation automorphism: $\tau_{(l, m)}\left(\xi_{i j}\right)=\xi_{i+l, j+m}$.

Theorem 2. (1) Let

$$
\begin{gathered}
\psi=\psi_{\beta}, \quad \delta=2\left(K_{1}^{*}-\left|K_{2}\right|\right) \quad \text { for } \quad|\beta|<\beta_{c}, \\
\psi=\psi_{\beta+} \text { or } \quad \psi_{\beta-}, \quad \delta=4\left(\left|K_{2}\right|-K_{1}^{*}\right) \quad \text { for } \quad|\beta|>\beta_{c},
\end{gathered}
$$

where $K_{1}=\beta J_{1}, K_{2}=\beta J_{2}$, and $K_{1}^{*}=(1 / 2) \log \operatorname{coth}\left|K_{1}\right| . \quad\left(\beta_{c}>0\right.$ is a solution of $K_{1}^{*}=\left|K_{2}\right|$.) Then

$$
\lim _{l \rightarrow \infty} e^{|l| \delta}\left|\psi\left(F_{1} \tau_{(l, 0)}\left(F_{2}\right)\right)-\psi\left(F_{1}\right) \psi\left(F_{2}\right)\right|=0
$$

for any local $F_{1}$ and $F_{2}$, and there exists $F_{\varepsilon 1}$ and $F_{\varepsilon 2}$ for any $\varepsilon>0$ such that

$$
\lim _{l \rightarrow \infty} e^{|l|(\delta+\varepsilon)}\left|\psi\left(F_{\varepsilon 1} \tau_{(l, 0)}\left(F_{\varepsilon 2}\right)\right)-\psi\left(F_{\varepsilon 1}\right) \psi\left(F_{\varepsilon 2}\right)\right|=\infty .
$$

(2) For any continuous functions $F_{1}$ and $F_{2}$ on the configuration space supported in $\left\{(l, m) \in \mathbb{Z}^{2} ; l \leqq N\right\}$ and $\left\{(l, m) \in \mathbb{Z}^{2} ; l \geqq N+d\right\}$, respectively,

$$
\left|\psi\left(F_{1} F_{2}\right)-\psi\left(F_{1}\right) \psi\left(F_{2}\right)\right| \leqq e^{-d \delta} \psi\left(\left|F_{1}\right|^{2}\right)^{1 / 2} \psi\left(\left|F_{2}\right|^{2}\right)^{1 / 2}
$$

(3) For any continuous functions $F_{1}$ and $F_{2}$ on the configuration space $\{1,-1\}^{\mathbb{Z}^{2}}$

$$
\lim _{(l, m) \rightarrow \infty} \psi\left(F_{1} \tau_{(l, m)}\left(F_{2}\right)\right)=\psi\left(F_{1}\right) \psi\left(F_{2}\right) .
$$

By the transfer matrix method, the state $\psi_{\beta}$ is related to a state $\varphi_{\beta}$ of a spin lattice system (of spin 1/2) in one-dimension by

$$
\psi_{\beta}(F)=\varphi_{\beta}\left(F_{\beta}\right),
$$

where $F$ is any-function of a finite number of $\xi$ 's and $F_{\beta}$ is a corresponding strictly local operator belonging to the $C^{*}$-algebra $\mathfrak{A}$ generated by Pauli spin matrices at all sites of the one-dimensional lattice $\mathbb{Z}$. [See Eqs. (4.4) and (4.5) of Sect. 4.] In [5], it has been shown that, for $|\beta|<\beta_{c}, \varphi_{\beta}$ is pure, while

$$
\varphi_{\beta}=\left(\varphi_{\beta+}+\varphi_{\beta-}\right) / 2
$$

for $|\beta|>\beta_{c}$ where states $\varphi_{\beta \pm}$ of $\mathfrak{A}$ give rise to disjoint representations of $\mathfrak{A}$. A key result is the following:

\section{Proposition 1.1.}

$$
\omega_{\beta \pm}(F)=\varphi_{\beta \pm}\left(F_{\beta}\right)
$$

defines states $\omega_{\beta \pm}$ on the abelian $C^{*}$-algebra generated by $\xi$ 's which are ergodic and mixing equilibrium states for the Hamiltonian (1.1). (Ergodicity and the mixing property refer to lattice translations as usual.) states:

Equations (1.5) and (1.7) imply the ergodic decomposition of equilibrium

$$
\psi_{\beta}=\left(\omega_{\beta+}+\omega_{\beta-}\right) / 2
$$


This much can be established without any outside information on $\psi_{\beta}$. However, we do not know at this point how to decide whether $\omega_{\beta \pm}$ are extremal equilibrium states. Thus we refer to the result of [10] that the decomposition (1.4) is an ergodic decomposition, to obtain the following identification.

Corollary 1.2. $\psi_{\beta \pm}=\omega_{\beta \pm}$.

We note that the labelling \pm of $\omega_{\beta \pm}$ for varying $\beta$ is fixed by analytic continuation except for the overall choice of the labelling. Since $\pm \psi_{\beta \pm}\left(\xi_{i j}\right)>0$, the analyticity of the quantity $\varphi_{\beta \pm}\left(\sigma_{z}^{(j)}\right)$ in $\beta$ then requires the labelling on two sides of Corollary 1.2 to be independent of $\beta\left(|\beta|>\beta_{c}\right)$. The over-all labelling of $\omega_{\beta \pm}$ is adjusted to coincide with that of $\psi_{\beta \pm}$.

We may also use the clustering property of $\psi_{\beta \pm}[10]$ to obtain an alternative direct proof of Corollary 1.2 (see Sect. 2). We are indebted to Dr. D. E. Evans for this direct and simple proof.

By using the same method as the case of the $X Y$ model [7], we prove the analytic dependence of $\varphi_{\beta \pm}$ on $\beta J_{1}$ and $\beta J_{2}$ :

Proposition 1.3. $\varphi_{\beta}(A)$ and $\varphi_{\beta \pm}(A)$ are real analytic as a function of $K_{1}=\beta J_{1}$ and $K_{2}=\beta J_{2}$ (hence as a function of $\beta$ ) except at $\beta=\beta_{c}$ for any strictly local $A \in \mathfrak{A}$.

As will be seen in Sect. $4, F_{\beta}$ is a linear combination of local observables with entire function of $\beta$ as coefficients. Equation (1.5) for $|\beta|<\beta_{c}$ and Corollary 1.2 for $|\beta|>\beta_{c}$ together with Proposition 1.3 prove our main Theorem 1 .

We present a proof of Proposition 1.1 in Sect. 2, a proof of Proposition 1.3 in Sect. 3 and the strict locality of $F_{\beta}$ along with a preparation for subsequent sections in Sect. 4.

The states $\varphi_{\beta}, \varphi_{\beta \pm}$ are actually ground states of $\mathfrak{U}$ with respect to a oneparameter group of automorphisms $\alpha_{t}^{\beta}$ of $\mathfrak{U}$ defined from the transfer matrix. For

$$
F=\prod_{j=1}^{n} \xi\left(k_{j}, I_{j}\right), \quad \xi\left(k_{j}, I_{j}\right)=\prod_{i \in I_{j}} \xi_{k_{j} i}
$$

with $k_{1}<k_{2}<\ldots<k_{n}$, the corresponding operator $F_{\beta} \in \mathfrak{U}$ is given by

$$
\begin{gathered}
F_{\beta}=\alpha_{i k_{1}}^{\beta}\left(\sigma_{x}\left(I_{1}\right)\right) \ldots \alpha_{i k_{n}}^{\beta}\left(\sigma_{x}\left(I_{n}\right)\right), \\
\sigma_{x}(I)=\prod_{i \in I} \sigma_{x}^{(i)} .
\end{gathered}
$$

Thus the relation (1.5) tells us that $\psi_{\beta}$ and $\psi_{\beta \pm}$ are obtained as Schwinger functions of ground states $\varphi_{\beta}$ and $\varphi_{\beta \pm}$. This view point is used (1) to prove the hermiticity $\omega_{\beta \pm}^{*}=\omega_{\beta \pm},(2)$ to prove Theorem 2 , and (3) to prove $\omega_{\beta+} \neq \omega_{\beta-}$ [without using the identification with $\psi_{\beta \pm}$ and without explicitly computing $\omega_{\beta \pm}(A)$ for some $\left.A\right]$.

The dynamical system $\left(\mathfrak{U}, \alpha_{t}^{\beta}\right)$ will be introduced and the relation $(1.10)$ will be established in Sect. 5, clustering properties will be proved in Sect. 6 and the last point $\omega_{\beta+} \neq \omega_{\beta}$ - will be discussed in Sect. 7. In the final Sect. 8, we use a conjugate automorphism $j$ of $\mathfrak{A}$, which is the time reversal symmetry in the sense $j \alpha_{t}=\alpha_{-t} j$, to deduce the hermiticity of $\omega_{\beta \pm}$. The same method has an application to the onedimensional XY-model, as is given in Proposition 8.6.

By the automorphism (of the classical configuration space), changing $\xi_{i, j}$ to $-\xi_{i, j}$ for $i$ odd while keeping $\xi_{i, j}$ for $i$ even unchanged, $J_{1}$ changes to $-J_{1}$ in $H$. A 
similar statement holds for $J_{2}$. By another automorphism changing $\xi_{i, j}$ to $\xi_{j, i}$ for all $(i, j) \in \mathbb{Z}^{2}, J_{1}$ and $J_{2}$ are interchanged. The equilibrium states depend on real parameters $K_{1}=\beta J_{1}$ and $K_{2}=\beta J_{2}$. Therefore, except for the point $K_{1}=K_{2}=0$, we may assume $K_{1}>0$, which means $K_{1}^{*}$ defined by (1.5) of [5] is real and positive. At the same time we may assume $-\varepsilon<K_{2}$ for any $\varepsilon>0$. (For analyticity at $K_{2}=0$, we have to consider slightly negative $K_{2}$, too.) The analyticity of correlation functions at $\beta=0$ is immediate, for example from the unique determination of the correlation functions by Kirkwood-Salzburg equations [11]. Thus the point $K_{1}=K_{2}=0$ is taken care of and we assume $K_{1}>0, K_{2}>-K_{1}^{*}$ in the following. We also assume $K_{2} \neq K_{1}^{*}$ (i.e. $\beta \neq \beta_{c}$ ), because we are proving analyticity and exponential clustering, which fail for $K_{2}=K_{1}^{*}$.

\section{From States of the Non-Commutative Algebra in One-Dimension to States of the Commutative Algebra in Two-Dimensions}

The map $F \rightarrow F_{\beta}$ will be studied in Sect. 4 and the following property will be basic for the present discussion.

Lemma 2.1. (1) $F_{\beta}$ is linear in $F$ and $\mathbf{1}_{\beta}=\mathbf{1}$.

(2) $F_{\beta}$ satisfies $\left(\tau_{(0, n)} F\right)_{\beta}=\tau_{n}\left(F_{\beta}\right)$ and the clustering property

$$
\lim _{n \rightarrow \infty}\left\|\left(F_{1} \tau_{(0, n)}\left(F_{2}\right)\right)_{\beta}-\left(F_{1}\right)_{\beta} \tau_{n}\left(\left(F_{2}\right)_{\beta}\right)\right\|=0,
$$

where $\tau_{(0, n)}$ is the lattice translation automorphism $\xi_{i j} \rightarrow \xi_{i, j+n}$ of the $C^{*}$-algebra $\mathbb{C}$ generated by $\xi_{i j}$ and $\tau_{n}$ is the lattice transformation automorphism of $\mathfrak{A}$.

(3) For the automorphism $\Theta$ of $\mathfrak{A}$ determined by (2.9) of [5] and the automorphism $\Theta$ of the abelian $C^{*}$-algebra $\mathfrak{C}$ generated by $\xi_{i j}$ determined by $\Theta\left(\xi_{i j}\right)=-\xi_{i j}$,

$$
(\Theta(F))_{\beta}=\Theta\left(F_{\beta}\right) .
$$

The states $\varphi_{\beta \pm}$ of $\mathfrak{A}$ are lattice translation invariant and pure [5]. Hence they have the clustering property

$$
\lim _{n \rightarrow \infty} \varphi_{\beta \pm}\left(A \tau_{n}(B)\right)=\varphi_{\beta \pm}(A) \varphi_{\beta \pm}(B) .
$$

We also use the result of Sect. 8 that $\varphi_{\beta \pm}\left(F_{\beta}\right)$ is real if $F$ is real (as a consequence of the " $j$-symmetry" of $\varphi_{\beta \pm}$ proved in Sect. 8).

Lemma 2.2. If $F \geqq 0$, then $\varphi_{\beta \pm}\left(F_{\beta}\right) \geqq 0$.

Proof. By (1.5), $F \geqq 0$ implies

$$
\varphi_{\beta+}\left(F_{\beta}\right)+\varphi_{\beta-}\left(F_{\beta}\right)=2 \varphi_{\beta}\left(F_{\beta}\right)=2 \psi_{\beta}(F) \geqq 0 .
$$

By (2.2), $\left(F_{1} \Theta\left(F_{2}\right)+\Theta\left(F_{1}\right) F_{2}\right)_{\beta}$ is $\Theta$ invariant. Since $F_{1} \geqq 0$ and $F_{2} \geqq 0$ imply $F_{1} \Theta\left(F_{2}\right)+\Theta\left(F_{1}\right) F_{2} \geqq 0$, we obtain

$$
\begin{aligned}
& \varphi_{\beta+}\left(\left(F_{1} \Theta\left(F_{2}\right)+\Theta\left(F_{1}\right) F_{2}\right)_{\beta}\right)+\varphi_{\beta-}\left(\left(F_{1} \Theta\left(F_{2}\right)+\Theta\left(F_{1}\right) F_{2}\right)_{\beta}\right) \\
& \quad=2 \psi_{\beta}\left(F_{1} \Theta\left(F_{2}\right)+\Theta\left(F_{1}\right) F_{2}\right) \geqq 0 .
\end{aligned}
$$


By (2.1), (2.3), and (2.5),

$$
\begin{aligned}
2 \varphi_{\beta+} & \left(F_{\beta}\right) \varphi_{\beta+}\left(\Theta\left(F_{\beta}\right)\right)+2 \varphi_{\beta-}\left(F_{\beta}\right) \varphi_{\beta-}\left(\Theta\left(F_{\beta}\right)\right) \\
= & \lim _{n \rightarrow \infty}\left\{\varphi_{\beta+}\left(\left(F \Theta\left(\tau_{(0, n)}(F)\right)+\Theta(F) \tau_{(0, n)}(F)\right)_{\beta}\right)\right. \\
& \left.+\varphi_{\beta-}\left(\left(F \Theta\left(\tau_{(0, n)}(F)\right)+\Theta(F) \tau_{(0, n)}(F)\right)_{\beta}\right)\right\} \geqq 0 .
\end{aligned}
$$

Since $\varphi_{\beta \pm} \Theta=\varphi_{\beta \mp}$, we obtain

$$
\varphi_{\beta+}\left(F_{\beta}\right) \varphi_{\beta-}\left(F_{\beta}\right) \geqq 0 .
$$

As quoted before the statement of the lemma, $\varphi_{\beta \pm}\left(F_{\beta}\right)$ is real for $F \geqq 0$. Combining (2.4) and (2.7), we obtain

$$
\omega_{\beta \pm}(F) \equiv \varphi_{\beta \pm}\left(F_{\beta}\right) \geqq 0
$$

for $F \geqq 0$. Q.E.D.

Proof of Proposition 1.1. Lemma 2.2 means that the restriction of $\omega_{\beta \pm}$ to the finite dimensional subalgebra $\mathfrak{C}(I)$ generated by $\xi_{i, j},(i, j) \in I$, for any finite subset $I$ are states [the linearity from Lemma 2.1 (1), the positivity from (2.8) and the normalization by $\omega_{\beta \pm}(\mathbf{1})=\varphi_{\beta \pm}(\mathbf{1})=1$ due to Lemma $\left.2.1(1)\right]$. Hence $\left\|\omega_{\beta \pm}\right\| \leqq 1$ and $\omega_{\beta \pm}$ extends to a state on the $C^{*}$ algebra $\mathfrak{C}$ generated by $\mathfrak{C}(I)$. (Alternatively, we may refer to the Kolmogorov theorem.)

By Lemma 2.1 (2) and the translational invariance of $\varphi_{\beta \pm}$,

$$
\omega_{\beta \pm}\left(\tau_{(0, n)} F\right)=\omega_{\beta \pm}(F) .
$$

Furthermore, by (2.1) and (2.3)

$$
\lim _{n \rightarrow \infty} \omega_{\beta \pm}\left(F_{1} \tau_{(0, n)} F_{2}\right)=\omega_{\beta \pm}\left(F_{1}\right) \omega_{\beta \pm}\left(F_{2}\right) .
$$

Going over to the cyclic (GNS) representation $\pi_{\beta \pm}$ on $\mathfrak{H}_{\beta \pm}, \tau_{(0, n)}$ will be represented by a one-parameter group of unitaries $U_{(0, n)}$, and $(2.10)$ means

$$
\lim _{n \rightarrow \infty}\left(\Psi, U_{(0, n)} \Phi\right)=\left(\Psi, \Omega_{\beta \pm}\right)\left(\Omega_{\beta \pm}, \Phi\right)
$$

for $\Psi, \Phi \in \mathfrak{H}_{\beta \pm}$, where $\Omega_{\beta \pm}$ is the canonical cyclic vector corresponding to $F=1$. [Equation (2.10) implies (2.11) for a dense set of vectors $\Psi$ and $\Phi$, which then implies (2.11) for general $\Psi$ and $\Phi$ by $L_{2}$ approximation.] This is the strong mixing property, which implies ergodicity (all relative to the group of one-dimensional lattice translation).

The mixing property relative to the two-dimensional translations will be shown later, but the properties relative to $\tau_{(0, n)}$ are sufficient for the proof of our main theorem indicated in Sect. 1.

Finally, we prove that $\omega_{\beta \pm}$ are equilibrium states. Because of the cluster property $(2.10)$, we have

$$
\underset{n \rightarrow \infty}{-\lim _{n \rightarrow \infty}} \tau_{(0, n)}(F)=\omega_{\beta \pm}(F) \mathbf{1}
$$

on $\mathfrak{S}_{\beta \pm}$ for each strictly local $F$. Since $\omega_{\beta}$ is a vector state by $2^{-1 / 2}\left(\Omega_{\beta+} \oplus \Omega_{\beta-}\right)$ on $\mathfrak{H}_{\beta} \equiv \mathfrak{H}_{\beta+} \oplus \mathfrak{S}_{\beta-}$, we have

$$
w_{n \rightarrow \infty} \lim _{(0, n)}(F)=\omega_{\beta+}(F) P_{+}+\omega_{\beta-}(F) P_{-},
$$


where $P_{ \pm}$are projections on $\mathfrak{G}_{\beta \pm}$. Thus (2.13) for all possible $F$ are observables at infinity according to Lanford and Ruelle [13] and induce the decomposition (1.8) (if $\omega_{\beta+} \neq \omega_{\beta-}$ ). By the result of [13], $\omega_{\beta \pm}$ are equilibrium states (i.e. satisfies the DLR equations). (Note that if $\omega_{\beta+}=\omega_{\beta-}$, then $\omega_{\beta \pm}=\psi_{\beta}$ is an equilibrium state. If this happens, $\psi_{\beta}$ would be ergodic and mixing. In the present model, this is not the case.) Q.E.D.

$A$ Direct Proof of Corollary 1.2. For any $\Theta$-even element $F, F_{\beta}$ is $\Theta$-even and

$$
\psi_{\beta \pm}(F)=\psi_{\beta}(F)=\varphi_{\beta}\left(F_{\beta}\right)=\varphi_{\beta \pm}\left(F_{\beta}\right) .
$$

Next we note that $\psi_{\beta+}$ (and $\psi_{\beta-}$ ) has a clustering property [10] and the state $\varphi_{\beta+}$ also has a clustering property by $\tau_{n}$-asymptotic abelian property of the algebra as it is pure. For $\Theta$-odd $F_{1}$ and $F_{2}$, we obtain

$$
\psi_{\beta+}\left(F_{1}\right) \psi_{\beta+}\left(F_{2}\right)=\varphi_{\beta+}\left(F_{1 \beta}\right) \varphi_{\beta+}\left(F_{2 \beta}\right)
$$

by the clustering properties of

$$
\psi_{\beta+}\left(G_{n}\right)=\psi_{\beta}\left(G_{n}\right)=\varphi_{\beta}\left(\left(G_{n}\right)_{\beta}\right)=\varphi_{\beta+}\left(\left(G_{n}\right)_{\beta}\right)
$$

for $G_{n}=F_{1} \tau_{(0, n)}\left(F_{2}\right)$ as $n \rightarrow \infty$. (2.15) with $F_{1}=F_{2}$ implies

$$
\psi_{\beta+}(F)= \pm \varphi_{\beta+}\left(F_{\beta}\right)
$$

with the sign \pm common for all $F$ again by (2.15). The labelling has been already discussed. Q.E.D.

In spite of this simple proof (due to D. E. Evans), we retained the argument via Proposition 2.1 as it shows which information on the two-dimensional classical lattice model can be obtained by working only with a one-dimensional quantum system, i.e. we can obtain an ergodic decomposition of $\psi_{\beta}$ into equilibrium states without any input on the property of $\psi_{\beta}$.

\section{Analyticity}

First we describe $\varphi_{\beta}$ in more detail, following [5]. We recall that an extension of $\mathfrak{A}$ to a larger $C^{*}$-algebra $\hat{\mathfrak{U}}=\mathfrak{U}+T \mathfrak{U}$ by an addition of a new element $T$ satisfying (2.1) of [6] (or (2.10) of [5]). The algebra $\hat{\mathfrak{A}}$ contains the Fermion algebra $\mathfrak{A}^{\mathrm{CAR}}$ on the one-dimensional lattice $\mathbb{Z}$. The involution automorphism $\Theta$ of $\mathfrak{A}$ is extended to the automorphism $\Theta$ of $\hat{\mathfrak{A}}$ (denoted by the same letter here) by $\Theta(T)=T$. Both $\mathfrak{A}$ and $\mathfrak{A}^{\mathrm{CAR}}$ are $\Theta$ invariant subalgebras of $\hat{\mathfrak{A}}$ with the following decompositions into $\Theta$-even and $\Theta$-odd parts:

$$
\mathfrak{U}=\mathfrak{U}_{+}+\mathfrak{U}_{-}, \quad \mathfrak{U}^{\mathrm{CAR}}=\mathfrak{U}_{+}^{\mathrm{CAR}}+\mathfrak{U}_{-}^{\mathrm{CAR}} .
$$

Then

$$
\mathfrak{H}_{+}=\mathfrak{U}_{+}^{\mathrm{CAR}}, \quad \mathfrak{U}_{-}=T \mathfrak{H}_{-}^{\mathrm{CAR}} .
$$

Strictly local elements of $\mathfrak{U}_{ \pm}$are exactly strictly local elements of $\mathfrak{Q}_{ \pm}^{\text {CAR }}$ except that $T$ is to be multiplied in case of $\Theta$-odd elements.

Annihilation and creation operators are denoted in a unified manner by $B(h)$, $h \in l_{2}(\mathbb{Z})+l_{2}(\mathbb{Z}) \equiv \Omega$ in the selfdual formalism of the canonical anticommutation 
relations (CAR) [3]. A Fock state $\varphi_{E}$ of $\mathfrak{A}^{\mathrm{CAR}}$ is specified by a projection operator $E$ on $\mathfrak{A}$ [satisfying $\Gamma E \Gamma+E=1$, where $B(h)^{*}=B(\Gamma h)$ ] through the relation $\varphi_{E}\left(B\left(h_{1}\right)^{*} B\left(h_{2}\right)\right)=\left(h_{1}, E h_{2}\right)$. The state $\varphi_{\beta}$ is a $\Theta$-invariant extension (to $\left.\mathfrak{A}\right)$ of the restriction of a Fock state $\varphi_{E}$ of $\mathfrak{A}^{\mathrm{CAR}}$ to $\mathfrak{A}_{+}^{\mathrm{CAR}}=\mathfrak{A}_{+}$, where $E$ is a multiplication of

$$
E(\theta)=(\mathbf{1}+V(\theta)) / 2
$$

on the Fourier transform of $\Omega=l_{2}(\mathbb{Z})+l_{2}(\mathbb{Z})$ with $\gamma(\theta)$ and $V(\theta)$ defined by (3.8) and (3.7) of [5].

From (3.8) of [5], $\gamma(\theta)$ is an analytic function of $K_{1}^{*}, K_{2}$, and $e^{i \theta}$, if $\sinh \gamma(\theta) \neq 0$, and so is $e^{i \delta(\theta)}$ by (3.9) of [5]. Therefore, $E(\theta)$ is an analytic function of $K_{1}^{*}, K_{2}$, and $e^{i \theta}$ if $K_{1}^{*}$ and $K_{2}$ are real and unequal (i.e. $\beta \neq \beta_{c}$ for real values of parameters).

The rest is exactly the same as [7]. Let $K_{1}^{*} \neq K_{2}$ and let $E^{\prime}$ be the projection operator corresponding to another value $\left(K_{1}^{\prime *}, K_{2}^{\prime}\right)$ in a sufficiently small neighbourhood of $\left(K_{1}^{*}, K_{2}\right)$. Let $U_{12}$ be given by (3.3) of [7] where $E_{1}$ and $E_{2}$ should now be replaced by $E$ and $E^{\prime}$. Then $U_{12}(\theta)$ is analytic in $K_{1}^{*}, K_{2}, K_{1}^{\prime *} K_{2}^{\prime}$, and $e^{i \theta}$. By Lemma 2 and (2.17) of [7] $U_{12} \theta_{-} U_{12}^{-1} \theta_{-}-1$ is in the trace class and with sufficiently small norm so that the Bogoliubov transformation $\alpha\left(U_{12}\right)$ of $\mathfrak{A}_{+}^{\text {CAR }}$ can be extended to a $*$-automorphism of $\hat{\alpha}\left(U_{12}\right)$ commuting with $\theta$ due to the Evans-Lewis criterion (see Lemma 1 of [7]) and we obtain

$$
\varphi_{\beta}^{\prime}(A)=\varphi_{\beta}\left(\hat{\alpha}\left(U_{12}\right) A\right)
$$

because $\varphi_{E^{\prime}}(A)=\varphi_{E}\left(\alpha\left(U_{12}\right)(A)\right)$ for $A \in \mathfrak{A}_{+}^{\mathrm{CAR}}=\mathfrak{A}_{+}$and $\varphi_{\beta}^{\prime}(A)=0=\varphi_{\beta}\left(\hat{\alpha}\left(U_{12}\right) A\right)$ for $A \in \mathfrak{A}_{-}$[and then $\hat{\alpha}\left(U_{12}\right) A \in \mathfrak{A}_{-}$] by definition of $\varphi_{\beta}^{\prime}$ and $\varphi_{\beta}$, where $\varphi_{\beta}^{\prime}$ corresponds to the parameters $K_{1}^{\prime *}, K_{2}^{\prime}$.

Defining $L$ by $U_{12} \theta_{-} U_{12}^{-1} \theta_{-}=e^{i L}$, with $\|L\|<\pi$, we see that $L$ is holomorphic in $\left(K_{1}^{\prime *}, K_{2}^{\prime}\right)$ (relative to the trace class norm) and hence $u=e^{i(B, L B) / 2}$ is holomorphic in $\left(K_{1}^{\prime \prime}, K_{2}^{\prime}\right)$ (relative to the operator norm), where $(B, L B)$ is the bilinear Hamiltonian. Thus $\hat{\alpha}\left(U_{12}\right)(T)=u T$ is holomorphic in $\left(K_{1}^{\prime *}, K_{2}^{\prime}\right)$. Since any strictly local observable $A$ in $\mathfrak{A}$ is a polynomial of $B(h)$ 's and $T$, the analyticity of $\alpha\left(U_{12}\right)(B(h))=B\left(U_{12} h\right)$ and $\hat{\alpha}\left(U_{12}\right)(T)$ just proved implies the same for $\hat{\alpha}\left(U_{12}\right) A$ and we obtain the analyticity of (3.4) and hence Proposition 1.3.

Remark. Equilibrium states $\psi_{\beta}$ of the Ising model can be related to a ground state of the $X Y$-model in one dimension (with a different definittion of $F_{\beta}$ ) [15]. Then the proof of this section can be entirely omitted by referring to results of [7]. We have not done so here because our results in Sect. 5 and later would require an introduction of $\alpha_{t}^{\beta}$ different from the $X Y$-model and different from [5], so that it would lengthen the paper.

\section{Strict Locality of $F_{\beta}$}

For $Q \in \hat{\mathfrak{A}}$, let

$$
\begin{gathered}
\alpha_{z}^{\beta 1 M}(Q)=\operatorname{Ad}\left[\exp \left(-i z K_{1}^{*} \sum_{j=-M}^{M} \sigma_{z}^{(j)}\right)\right](Q), \\
\alpha_{z}^{\beta 2 M}(Q)=\operatorname{Ad}\left[\exp \left(-i z K_{2} \sum_{j=-M}^{M-1} \sigma_{x}^{(j)} \sigma_{x}^{(j+1)} / 2\right)\right](Q),
\end{gathered}
$$


where $\operatorname{Ad}[V](Q)=V Q V^{-1}$. Then

$$
\alpha_{i}^{\beta M}(Q) \equiv T_{M} Q T_{M}^{-1}=\alpha_{i}^{\beta 2 M} \alpha_{i}^{\beta 1 M} \alpha_{i}^{\beta 2 M}(Q),
$$

where $T_{M}$ is the transfer matrix given by (1.4) of [5].

Any polynomial of a finite number of $\xi_{i j}$ is a linear combination of the monomials $F$ of $\xi$ 's given by (1.12). The corresponding element $F_{\beta} \in \mathfrak{U}$ is defined as follows:

$$
\begin{gathered}
F_{\beta}=\lim _{M \rightarrow \infty} F_{\beta M}, \\
F_{\beta M}=T_{M}^{k_{1}} \sigma_{x}\left(I_{1}\right) T_{M}^{k_{2}-k_{1}} \sigma_{x}\left(I_{2}\right) T_{M}^{k_{3}-k_{2}} \ldots \sigma_{x}\left(I_{n}\right) T_{M}^{-k_{n}} \\
=\left\{\left(\alpha_{i}^{\beta M}\right)^{k_{1}} \sigma_{x}\left(I_{1}\right)\right\}\left\{\left(\alpha_{i}^{\beta M}\right)^{k_{2}} \sigma_{x}\left(I_{2}\right)\right\} \ldots\left\{\left(\alpha_{i}^{\beta M}\right)^{k_{n}} \sigma_{x}\left(I_{n}\right)\right\} .
\end{gathered}
$$

We denote the subalgebra of $\mathfrak{A}$ generated by $\sigma_{\alpha}^{(j)}$ with $|j| \leqq n$ by $\mathfrak{A}_{n}$. The following lemma shows the strict locality of $F_{\beta}$.

Lemma 4.1. For $Q \in \mathfrak{U}_{n}$,

$$
\begin{gathered}
\alpha_{z}^{\beta 1}(Q) \equiv \lim _{M \rightarrow \infty} \alpha_{z}^{\beta 1 M}(Q)=\alpha_{z}^{\beta 1 n}(Q) \in \mathfrak{A}_{n}, \\
\alpha_{z}^{\beta 2}(Q) \equiv \lim _{M \rightarrow \infty} \alpha_{z}^{\beta 2 M}(Q)=\alpha_{z}^{\beta 2(n+1)}(Q) \in \mathfrak{A}_{n+1} .
\end{gathered}
$$

Proof. By commutativity of summands, we obtain

$$
\begin{aligned}
\exp \left(-i z K_{1}^{*} \sum_{j} \sigma_{z}^{(j)}\right) & =\prod_{j} \exp \left(-i z K_{1}^{*} \sigma_{z}^{(j)}\right), \\
\exp \left(-i z K_{2} \sum_{j} \sigma_{x}^{(j)} \sigma_{x}^{(j+1)}\right) & =\prod_{j} \exp \left(-i z K_{2} \sigma_{x}^{(j)} \sigma_{x}^{(j+1)}\right) .
\end{aligned}
$$

By commutativity of $Q \in \mathfrak{U}_{n}$ and the factors in (4.8) with $j>n,(4.6)$ follows from (4.8). Similarly (4.7) follows from (4.9). Q.E.D.

By (4.6) and (4.7), $F_{\beta}$ can be explicitly calculated as a polynomial of $\sigma$ 's with analytic functions of $K$ 's as coefficients by

$$
\begin{aligned}
\exp \left(-i z K_{1}^{*} \sigma_{z}^{(j)}\right) & =\cosh z K_{1}^{*}-i \sigma_{z}^{(j)} \sinh z K_{1}^{*} \\
\exp \left(-i z K_{2} \sigma_{x}^{(j)} \sigma_{x}^{(j+1)}\right) & =\cosh z K_{2}-i \sigma_{x}^{(j)} \sigma_{x}^{(j+1)} \sinh z K_{2} .
\end{aligned}
$$

Corollary 4.2.

$$
\begin{gathered}
\alpha^{\beta}(Q) \equiv \lim _{M \rightarrow \infty} \alpha_{i}^{\beta M}(Q)=\alpha_{i}^{\beta 2} \alpha_{i}^{\beta 1} \alpha_{i}^{\beta 2}(Q), \\
F_{\beta}=\left\{\left(\alpha^{\beta}\right)^{k_{1}} \sigma_{x}\left(I_{1}\right)\right\}\left\{\left(\alpha^{\beta}\right)^{k_{2}} \sigma_{x}\left(I_{2}\right)\right\} \ldots\left\{\left(\alpha^{\beta}\right)^{k_{n}} \sigma_{x}\left(I_{n}\right)\right\} .
\end{gathered}
$$

Proof of Lemma 2.1. (1) Since monomials (1.9) are linearly independent, the linearity holds by definition.

(2) From the proof of Lemma 4.1, it immediately follows that $\tau_{n}$ commutes with $\alpha_{z}^{\beta 1}$ and $\alpha_{z}^{\beta 2}$. Hence $\left(\tau_{(0, n)} F\right)_{\beta}=\tau_{n}\left(F_{\beta}\right)$.

From the proof of Lemma 4.1, it is also clear that for sufficiently large $n$ (and local $F^{\prime}$ s), $\left(F_{1} \tau_{(0, n)}\left(F_{2}\right)\right)_{\beta}=\left(F_{1}\right)_{\beta}\left(\tau_{(0, n)}\left(F_{2}\right)\right)_{\beta}$. Therefore, $(2.1)$ follows. 
(3) From (4.1) and (4.2), $\Theta$ (in one-diemnsion) commutes with $\alpha_{z}^{\beta 1 M}$ and $\alpha_{z}^{\beta 2 M}$. Thus, for $F$ given by (1.12),

$$
(\Theta F)_{\beta M}=\left\{\left(\alpha_{i}^{\beta M}\right)^{k_{1}} \Theta \sigma_{x}\left(I_{1}\right)\right\} \ldots\left\{\left(\alpha_{i}^{\beta M}\right)^{k_{n}} \Theta \sigma_{x}\left(I_{n}\right)\right\}=\Theta\left(F_{\beta M}\right) .
$$

By taking limit $M \rightarrow \infty$, we obtain (2.2). Q.E.D.

\section{The Dynamical System $\left(\mathfrak{A r}, \alpha_{t}^{\beta}\right)$}

The aim of this section is to define a dynamical system $\left(\mathfrak{A}, \alpha_{t}^{\beta}\right)$ for the onedimensional lattice spin system, identify $\varphi_{\beta}$ and $\varphi_{\beta \pm}$ as ground states of this dynamical system and identify the quantities $\varphi_{\beta}\left(F_{\beta}\right)$ and $\varphi_{\beta \pm}\left(F_{\beta}\right)$ appearing in (1.5) and (2.7) as their Schwinger functions. For this purpose, we first introduce a dynamical system $\left(\mathfrak{U}^{\mathrm{CAR}}, \alpha_{t}^{\mathrm{CAR}}\right)$ and then extend it to $\left(\hat{\mathfrak{U}}, \hat{\alpha}_{t}^{\beta}\right)$ so that as its restriction to $\mathfrak{U} \subset \hat{\mathfrak{A}}$ we obtain the desired dynamical system $\left(\mathfrak{U}, \alpha_{t}^{\beta}\right)$.

Let $T_{M}$ be the transfer matrix given by (1.4) of [5] and used in the preceding section. We define

$$
\alpha_{z}^{\beta M}(A)=T_{M}^{-i z} A T_{M}^{i z}, \quad A \in \hat{\mathfrak{A}},
$$

which is consistent with notation $\alpha_{i}^{\beta M}$ of (4.3). Since $T_{M} \in \mathfrak{A}_{+}=\mathfrak{A}_{+}^{\mathrm{CAR}}, \alpha_{z}^{\beta M}$ leaves $\mathfrak{A}^{\mathrm{CAR}}$ invariant. Its restriction to $\mathfrak{U}^{\mathrm{CAR}}$ is determined by

$$
\alpha_{z}^{\beta M}(B(h))=B\left(e^{-2 i z H^{M}} h\right)
$$

which follows from the expression (2.23) of [5] for $T_{M}$ up to a constant factor. Here $H^{M}$ is defined by (2.22) of [5] and tends to $H$ defined by (3.5) of [5] in the strong operator topology. Hence

$$
\lim _{M \rightarrow \infty} \alpha_{z}^{\beta M}(B(h))=B\left(e^{-2 i z H} h\right),
$$

where the limit is in the norm topology of $\mathfrak{U}^{\mathrm{CAR}}$. Therefore,

$$
\alpha_{t}^{\mathrm{CAR}}(A) \equiv \lim _{M \rightarrow \infty} \alpha_{t}^{\beta M}(A), \quad A \in \mathfrak{U}^{\mathrm{CAR}}
$$

exists for real $t$ and defines a dynamical system $\left(\mathfrak{H}^{\mathrm{CAR}}, \alpha_{t}^{\mathrm{CAR}}\right)$.

Proposition 5.1. (1) The action $t \in \mathbb{R} \rightarrow \alpha_{t}^{\mathrm{CAR}} \in \mathrm{Aut}^{\mathrm{CAR}}$ extends to an action $t \in \mathbb{R}$ $\rightarrow \hat{\alpha}_{t}^{\beta} \in$ Aut $\hat{\mathfrak{A}}$.

(2) The extension $\hat{\alpha}_{t}^{\beta}$ of $\alpha_{t}^{\mathrm{CAR}}$ to $\hat{\mathfrak{U}}$ continuous in $t$ is unique.

(3) For any strictly local $A$ in $\hat{\mathfrak{U}}$ (i.e. $A=A_{1}+T A_{2}$, where $A_{1}$ and $A_{2}$ are strictly local elements of $\mathfrak{U}^{\mathrm{CAR}}$ (equivalently of $\left.\mathfrak{U}\right)$ ), $\hat{\alpha}_{t}^{\beta}(A)$ is entire analytic in $t$.

Remark 5.2. We are avoiding the question of whether (5.1) has a limit for $A \in \mathfrak{U}$ as $M \rightarrow \infty$.

Proof. (1) We will use the criterion of Evans and Lewis [9] that if the automorphism $\alpha_{t}^{\mathrm{CAR}} \Theta_{-} \alpha_{-t}^{\mathrm{CAR}} \Theta_{-}$of $\mathfrak{U}^{\mathrm{CAR}}$ is implementable by a unitary $u_{t}$ in $\mathfrak{X}_{+}^{\mathrm{CAR}}$

$$
\operatorname{Ad} u_{t}=\alpha_{t}^{\mathrm{CAR}} \Theta_{-} \alpha_{-t}^{\mathrm{CAR}} \Theta_{-},
$$

then $u_{t}$ can be chosen to satisfy

$$
u_{t} \Theta_{-}\left(u_{t}\right)=1
$$


and $\hat{\alpha}_{t}^{\beta}$ defined by

$$
\hat{\alpha}_{t}^{\beta}\left(A_{1}+T A_{2}\right)=\alpha_{t}^{\beta}\left(A_{1}\right)+u_{t} T \alpha_{t}^{\beta}\left(A_{2}\right)
$$

is an automorphism of $\hat{\mathfrak{A}}$ coinciding with $\alpha_{t}^{\mathrm{CAR}}$ on $\mathfrak{U}^{\mathrm{CAR}}$ (Lemma 1 of [7]).

Let $\alpha(U)$ denote the Bogoliubov automorphism of $\mathfrak{A}^{\mathrm{CAR}}$ such that $\alpha(U)(B(h))$ $=B(U h)$. Then $\alpha_{t}^{\mathrm{CAR}}=\alpha\left(e^{-2 i t H}\right)$ by (5.3) and

$$
\alpha_{t}^{\mathrm{CAR}} \Theta_{-} \alpha_{-t}^{\mathrm{CAR}} \Theta_{-}=\alpha\left(e^{-2 i t H} \theta_{-} e^{2 i t H} \theta_{-}\right) .
$$

After the Fourier transform of the test function space $K=l_{2}(\mathbb{Z}) \oplus l_{2}(\mathbb{Z}), H$ is a multiplication operator of the function

$$
H(\theta)=\gamma(\theta)(1-2 E(\theta))
$$

which is a holomorphic function of $z=e^{i \theta}$ at the unit circle $|z|=1$ if $|\beta| \neq \beta_{c}$ as indicated in Sect. 3. By Lemma 2 and (2.17) of [7] again, $e^{-2 i t H} \theta_{-} e^{2 i t H} \theta_{-}-1$ is in the trace class. For a Bogoliubov transformation $U_{t}=e^{-2 i t H} \theta_{-} e^{2 i t H} \theta_{-}$, its determinant is 1 or -1 depending on the even-oddness of the multiplicity of its eigenvalue -1 (due to $\Gamma U_{t} \Gamma=U_{t}$ ). Since $U_{t}$ is continuous in the norm topology and since $U_{t}-\mathbf{1}$ is compact, this is constant in $t$ and hence must be 1 due to $U_{t}=\mathbf{1}$ for $t=0$. Therefore, the criterion of Evans and Lewis is satisfied, and $\alpha_{t}$ for each $t$ can be extended to $\hat{\alpha}_{t} \in$ Aut $\hat{\mathfrak{A}}$.

We finish the proof of (1) by showing that $\hat{\alpha}_{t}$ can be chosen to be an action (of $t \in \mathbb{R}$ ). We first prove that $\hat{\alpha}_{t}$ can be chosen to be continuous in $t$, then we prove that such $\hat{\alpha}_{t}$ is automatically a one-parameter group.

By the same argument as above, the operator

$$
\Delta=\theta_{-} H \theta_{-}-H=-2\left(q_{+} H q_{-}+q_{-} H q_{+}\right)
$$

is in the trace class $\left(q_{ \pm}=\left(1 \pm \theta_{-}\right) / 2\right)$ if $\beta \neq \beta_{c}$. Therefore,

$$
U_{t}=1+2 i \int_{0}^{t} e^{-2 i s H} \Delta e^{2 i s \theta-H \theta-} d s
$$

is continuous in $t$ relative to the trace class norm. For sufficiently small $\delta>0$, $\left\|U_{t}-\mathbf{1}\right\|<1$ for $|t| \leqq \delta$. Then

$$
U_{t}=e^{i L_{t}}, \quad L_{t}\left(=\log \left(\mathbf{1}+U_{t}-\mathbf{1}\right)\right)=\sum_{n=1}^{\infty}(-1)^{n-1}\left(U_{t}-\mathbf{1}\right)^{n} / n
$$

where $L_{t}$ is continuous in $t$ relative to the trace class norm. Therefore, the choice $u_{t}=e^{i\left(B, L_{t} B\right) / 2}$ is continuous in $t$ and satisfies (5.5) (by (8.23) and (8.24) of [3]) as well as (5.6) (by $\Theta_{-}\left(B, L_{t} B\right)=\left(B, \theta_{-} L_{t} \theta_{-} B\right)$ due to (7.12) of [3] and by $\theta_{-} L_{t} \theta_{-}=-L_{t}$ due to $\left.\theta_{-} U_{t} \theta_{-}=U_{t}^{-1}\right)$, where $\left(B, L_{t} B\right)$ is the bilinear Hamiltonian and the continuity of $L_{t} \rightarrow\left(B, L_{t} B\right)$ is by (7.11) of [3]. For larger values of $t$, we define $u_{t}$ by the following cocycle equation:

$$
u_{t_{1}+\ldots+t_{n}}=\alpha_{t_{1}+\ldots+t_{n-1}}^{\beta}\left(u_{t_{n}}\right) \ldots \alpha_{t_{1}}^{\beta}\left(u_{t_{2}}\right) u_{t_{1}} .
$$

For example for $(n-1) \delta<|t| \leqq n \delta$, we use $t_{1}=\ldots=t_{n-1}=(\operatorname{sign} t) \delta$ and $t_{n}=t-t_{1}-\ldots-t_{n-1}$. Then $u_{t}$ so defined is continuous in $t \in \mathbb{R}$. Such $u_{t}$ automatically satisfies (5.5) [by (5.5) for $u_{t_{j}}$ ] and (5.6) [by $\Theta_{-}\left(u_{t_{j}}\right)=u_{t_{j}}^{-1}$ and by $\left.\operatorname{Ad}\left(u_{t_{j}}\right) \Theta_{-} \alpha_{t_{j}}^{\beta} \Theta_{-}=\alpha_{t_{j}}^{\beta}\right]$. Then the group property $\hat{\alpha}_{t_{1}}^{\beta} \hat{\alpha}_{t_{2}}^{\beta}=\hat{\alpha}_{t_{1}+t_{2}}^{\beta}$ follows from the uniqueness argument, i.e. the proof of (2), which will be presented now.

(2) For the proof of this part as well as for a later use, we need the following lemma to be proved immediately after the present proof. 
Lemma 5.3. Any element of $\hat{\mathfrak{U}}$ commuting with all elements of $\mathfrak{U}_{+}=\mathfrak{U}_{+}^{\mathrm{CAR}}$ must be a multiple of the identity operator.

Let $\hat{\alpha}_{t}^{\prime}$ be another extension of $\alpha_{t}$. Then $\hat{\alpha}_{t}^{\prime}(T) T \equiv u_{t}^{\prime}$ also has the property (5.5) and hence $u_{t}^{\prime} u_{t}^{*}$ commutes with all elements of $\mathfrak{U}^{\text {CAR }}$. By the above lemma we have $u_{t}^{\prime}=c_{t} u_{t}$ for a complex number $c_{t}$. Since $u_{t}^{\prime}$ and $u_{t}$ are unitary, $\left|c_{t}\right|=1$. Since $\hat{\alpha}_{t}^{\prime}(T)$ $=u_{t}^{\prime} T=c_{t} \hat{\alpha}_{t}(T)$, and since both $\hat{\alpha}_{t}^{\prime}(T)$ and $\hat{\alpha}_{t}(T)$ are selfadjoint, $c_{t}= \pm 1$. By the continuity in $t, c_{t}=1$ and we obtain the uniqueness.

(3) By (5.3), $\hat{\alpha}_{t}(A)$ is entire analytic for any polynomial $A$ of $B(h)$ 's. Therefore, we have only to prove the entire analyticity of $\hat{\alpha}_{t}(T)=u_{t} T$ or equivalently that of $u_{t}$.

Using the trace class operator $\Delta$ of $(5.10)$, we define

$$
\tilde{\Delta}=(B, \Delta B) \text {. }
$$

It is a selfadjoint element of $\mathfrak{U}_{+}^{\mathrm{CAR}}=\mathfrak{U}_{+}$and satisfies

$$
[\tilde{\Delta}, B(h)]=B(2 \Delta h)
$$

by (7.9) and (7.1) of [3] due to $\Gamma \Delta \Gamma=-\Delta\left(=-\Delta^{*}\right)$. By (7.12) of [3],

$$
\tilde{\Delta}(t) \equiv \alpha_{t}^{\beta}(\tilde{\Delta})=\left(B, e^{-2 i t H} \Delta e^{2 i t H} B\right)
$$

is entire analytic in $t$ as $\Delta(z) \equiv e^{-2 i z H} \Delta e^{2 i t H}$ is an entire function of $z$ with values in the trace class operators (cf. (7.11) of [3]).

We define

$$
v_{z}=\mathbf{1}+\sum_{n=1}^{\infty}(i z)^{n} \int_{0}^{1} d t_{1} \ldots \int_{0}^{t_{n}-1} d t_{n} \tilde{\Delta}\left(t_{1} z\right) \ldots \widetilde{\Delta}\left(t_{n} z\right)
$$

(see $\operatorname{Exp}_{l}$ defined in [4]). For $z=t$ real, $v_{t}$ is unitary by (2.14), (2.15), (2.17), and (2.18) of [4]. Furthermore, Proposition 12 of [4] implies that $\beta_{t}=\left(\operatorname{Ad} v_{t}^{*}\right) \alpha_{t}^{\mathrm{CAR}}$ is an automorphism of $\mathfrak{U}^{\mathrm{CAR}}$

$$
\left(v_{t}^{*}=\left(\operatorname{Exp}_{l}\left(\int_{0}^{t} ; i \tilde{\Delta}(t) d t\right)\right)^{*}=\operatorname{Exp}_{r}\left(\int_{0}^{t} ;-i \tilde{\Delta}(t) d t\right)\right.
$$

in the notation of [4]) with the generator given by

$$
\begin{aligned}
\frac{d}{d t} \beta_{t}(B(h)) & =\beta_{t}\left[\frac{d}{d s} \beta_{s}(B(h))\right]_{s=0} \\
& =\beta_{t}\left\{\frac{d}{d s} \alpha_{s}^{\mathrm{CAR}}(B(h))-i[\tilde{\Delta}, B(h)]\right\}_{s=0} \\
& =\beta_{t}(B(-2 i(H+\Delta) h))=\beta_{t}\left(B\left(-2 i \theta_{-} H \theta_{-} h\right)\right) .
\end{aligned}
$$

This implies that $\beta_{t}\left(B\left(e^{2 i \theta-H \theta-t} h\right)\right)$ has an identically vanishing $t$-derivative and hence is equal to its value $B(h)$ for $t=0$. Hence

$$
\begin{aligned}
\left(\operatorname{Ad} v_{t}\right)(B(h)) & =\left(\operatorname{Ad} v_{t}\right) \beta_{t}\left(B\left(e^{2 i \theta_{-} H \theta_{-} t} h\right)\right) \\
& =\alpha_{t}^{\mathrm{CAR}}\left(B\left(\theta_{-} e^{2 i H t} \theta_{-} h\right)=\alpha_{t}^{\mathrm{CAR}} \Theta_{-} \alpha_{-t}^{\mathrm{CAR}} \Theta_{-}(B(h))\right. \\
& =\left(\operatorname{Ad} u_{t}\right)(B(h)) .
\end{aligned}
$$

This implies the same equality with $B(h)$ replaced by an arbitrary element of $\mathfrak{A}^{\text {CAR }}$. 
As we have seen, (5.17) or (5.18) implies $\beta_{t}=\Theta_{-} \alpha_{t}^{\mathrm{CAR}} \Theta_{-}$. Since $\theta_{-} \Delta \theta_{-}=-\Delta$ by (5.10), we have $\Theta_{-}(\tilde{\Delta})=-\tilde{\Delta}$. Therefore,

$$
\begin{aligned}
\Theta_{-}\left(v_{t}\right) & =\operatorname{Exp}_{l}\left(\int_{0}^{t} ; \beta_{t}(-i \tilde{\Delta}) d t\right) \\
& =\operatorname{Exp}_{l}\left(\int_{0}^{t} ;((-i \tilde{\Delta}) *(-i \tilde{\Delta}))(s) d s\right)
\end{aligned}
$$

by definition (3.7) of [4] because $v_{t}^{*}=\operatorname{Exp}_{r}\left(\int_{0}^{t} ;-i \tilde{\Delta}(t) d t\right)$. By (3.11) of [4], $v_{t} \Theta_{-}\left(v_{t}\right)=1$. Since $v_{t}$ is continuous in $t$, we conclude by the uniqueness proof of $(2)$ that

$$
u_{t}=v_{t}
$$

For $|z| \leqq \delta,\|\tilde{\Delta}(t z)\| \leqq e^{4 \delta\|H\|}\|\Delta\|_{\text {tr }}$ for $|t| \leqq 1$ by (7.11) of [3], where we have $\Gamma \tilde{\Delta}(t z)^{*} \Gamma=-\tilde{\Delta}(t z)$ due to $\Gamma H^{*} \Gamma=-H$ and $\Gamma \Delta^{*} \Gamma=-\Delta$. Therefore,

$$
\begin{aligned}
& \left\|(i z)^{n} \int_{0}^{1} d t_{1} \ldots \int_{0}^{t_{n}-1} d t_{n} \tilde{\Delta}\left(t_{1} z\right) \ldots \tilde{\Delta}\left(t_{n} z\right)\right\| \\
& \leqq(n !)^{-1}\left(|z|\|\Delta\|_{\mathrm{tr}} e^{4 \delta\|\boldsymbol{H}\|}\right)^{n}
\end{aligned}
$$

and the sum in (5.16) is uniformly and absolutely convergent. By the entire analyticity of $\tilde{\Delta}(t), u_{t}=v_{t}$ given by (5.16) is entire analytic in $t$. Q.E.D.

Proof of Lemma 5.3. Let $Q \in \hat{\mathfrak{A}}$ commute with all elements of $\mathfrak{A}_{+}$. We have

$$
\Theta(Q)=\lim _{M \rightarrow \infty}\left(\prod_{j=-M}^{M} \sigma_{z}^{(j)}\right) Q\left(\prod_{j=-M}^{M} \sigma_{z}^{(j)}\right)=Q
$$

because $\left(\prod_{j=-M}^{M} \sigma_{z}^{j}\right)$ belongs to $\mathfrak{U}_{+}$and its square is 1 . Let $\widetilde{\Theta}_{-}$be the automorphism of $\hat{\mathfrak{A}}$ satisfying

$$
\tilde{\Theta}_{-}\left(A_{1}+A_{2} T\right)=A_{1}-A_{2} T
$$

for $A_{1}, A_{2} \in \mathfrak{A}$. (The dual automorphism of $\Theta_{-} \in$ Aut $\mathfrak{A}$.) Then

$$
\tilde{\Theta}_{-}(X)=\lim _{M \rightarrow \infty} \sigma_{x}^{(M)} \sigma_{x}^{(-M)} X \sigma_{x}^{(-M)} \sigma_{x}^{(M)}, \quad X \in \hat{\mathfrak{U}},
$$

due to $\lim _{j \rightarrow \infty}\left[\sigma_{x}^{(j)}, A\right]=0$ for $A \in \mathfrak{U}, \sigma_{x}^{(M)} T \sigma_{x}^{(M)}=T$ and $\sigma_{x}^{(-M)} T \sigma_{x}^{(-M)}=-T$ for $M>0$. Since $\sigma_{x}^{(M)} \sigma_{x}^{(-M)} \in \mathfrak{A}_{+}$, we have

$$
\tilde{\Theta}_{-}(Q)=Q \text {. }
$$

The two equations (5.22) and (5.25) imply $Q \in \mathfrak{U}_{+}$. Since $\mathfrak{U}_{+}=\mathfrak{A}_{+}^{\text {CAR }}$ is known to have a trivial center, we have $Q=c 1$. Q.E.D.

Remark 5.4. Lemma 5.3 implies that any unitary $u \in \hat{\mathfrak{A}}$ satisfying $u \mathfrak{U}_{+} u^{*}=\mathfrak{U}_{+}$ must belong to one of $\mathfrak{U}_{+}, T \mathfrak{U}_{+}, \mathfrak{U}_{-}$, and $T \mathfrak{U}_{-}$, because $u \Theta\left(u^{*}\right)$ and $u \tilde{\Theta}\left(u^{*}\right)$ commute with all elements of $\mathfrak{U}_{+}$and hence are multiples of identity. 
We now want to identify the analytic continuation of $\alpha_{z}^{\beta}(A)$ to $z=i$ with $\alpha^{\beta}(A)$ defined by

$$
\alpha^{\beta}(A)=\lim _{M \rightarrow \infty} T_{M} A T_{M}^{-1}=\alpha_{i}^{\beta 2} \alpha_{i}^{\beta 1} \alpha_{i}^{\beta 2}(A),
$$

where $A$ is a strictly local element of $\mathfrak{A}$, and $\alpha_{z}^{\beta j}(z=i$ here $), j=1,2$, are defined in Lemma 4.1.

Proposition 5.5. For any strictly local $A \in \mathfrak{U}$,

$$
\alpha_{i}^{\beta}(A)=\alpha^{\beta}(A) .
$$

Remark 5.6. For $A \in \mathfrak{U}^{\mathrm{CAR}}, \alpha_{z}^{\beta}(A)$ (including the case $z=i$ ) is defined by the same type of limit as (5.26). However, this is not the case for $A \in \mathfrak{A}$ (or equivalently for $A=T$ ) and we present a proof avoiding the discussion of such a limit in the following.

Proof. Let $\mathfrak{A}_{(0)}$ be the algebra of all strictly local elements of $\mathfrak{U}$ and $\mathfrak{B}$ be a subalgebra of $\hat{\mathfrak{A}}$ generated by $\mathfrak{U}_{(0)}$ and $T$ or equivalently by $T$ and $B(h)$ with strictly local $h$ 's. We now extend $\alpha_{z}^{\beta j}$ defined on $\mathfrak{A}_{(0)}$ by Lemma 4.1 to $\mathfrak{B}$ :

$$
\begin{aligned}
\alpha_{z}^{\beta 1}(B(h)) & =\lim \alpha_{z}^{\beta 1 M}(B(h))=\lim B\left(e^{-2 i z K_{1}^{*} H_{1}^{M}} h\right) \\
& =B\left(e^{-2 i z K_{1}^{*} H_{1}} h\right), \\
\alpha_{z}^{\beta 2}(B(h)) & =\lim \alpha_{z}^{\beta 2 M}(B(h))=\lim B\left(e^{-i z K_{2} H_{2}^{M}} h\right) \\
& =B\left(e^{-i z K_{2} H_{2}} h\right) .
\end{aligned}
$$

In view of (3.5) of [5] and (5.2) in this section, we obtain

$$
\alpha_{i}^{\beta}(A)=\alpha_{i}^{\beta 2} \alpha_{i}^{\beta 1} \alpha_{i}^{\beta 2}(A)
$$

for $A=B(h)$. For $h \in l_{2}(\mathbb{Z}) \oplus l_{2}(\mathbb{Z})$ with components $(h)_{j}=\delta_{1 j}\left(\begin{array}{l}1 \\ 1\end{array}\right), B(h)=c_{1}+c_{1}^{*}$ $=T \sigma_{x}^{(1)}$. Hence $T=B(h) \sigma_{x}^{(1)}$ for such $h$. Thus $\alpha_{i}^{\beta 2} \alpha_{i}^{\beta 1} \alpha_{i}^{\beta 2}(T)$ is well-defined. Let

$$
w \equiv \alpha_{i}^{\beta 2} \alpha_{i}^{\beta 1} \alpha_{i}^{\beta 2}(T) T \in \mathfrak{U}_{+} .
$$

Since $T^{2}=1, w^{-1}=T \alpha_{i}^{\beta 2} \alpha_{i}^{\beta 1} \alpha_{i}^{\beta 2}(T)$. We compute

$$
\begin{aligned}
w B(h) w^{-1} & =\alpha_{i}^{\beta 2} \alpha_{i}^{\beta 1} \alpha_{i}^{\beta 2}\left(T\left\{\left(\alpha_{-i}^{\beta 2} \alpha_{-i}^{\beta 1} \alpha_{-i}^{\beta 2}\right)(T B(h) T)\right\} T\right) \\
& =B\left(e^{K_{2} H_{2}} e^{2 K_{1}^{*} H_{1}} e^{K_{2} H_{2}} \theta_{-} e^{-K_{2} H_{2}} e^{-2 K_{1}^{*} H_{1}} e^{-K_{2} H_{2}} \theta_{-} h\right) \\
& =B\left(e^{2 H_{1}} \theta_{-} e^{-2 H_{1}} \theta_{-} h\right)=v_{i} B(h) v_{i}^{-1},
\end{aligned}
$$

where we obtain the last equality by the analytic continuation of

$$
v_{z} B(h)=B\left(e^{-2 i z H_{1}} \theta_{-} e^{2 i z H} \theta_{-} h\right) v_{z}
$$

from $z=t$ real to $z=i$. Therefore, $w^{-1} v_{i} \in \mathfrak{A}_{+}=\mathfrak{A}_{+}^{\text {CAR }}$ commutes with all $B(h)$ and hence is a multiple of the identity, for example by the simplicity of $\mathfrak{U}^{\text {CAR }}$ (or by Lemma 5.3):

$$
w=c v_{i} \text {. }
$$

Since $v_{z} \Theta_{-}\left(v_{z}\right)=\mathbf{1}$ and $\Theta_{-}\left(v_{z}\right)=T v_{z} T$, we have $\left(v_{z} T\right)^{2}=v_{z} T v_{z} T=v_{z} \Theta_{-}\left(v_{z}\right)=\mathbf{1}$. We also have $(w T)^{2}=\alpha_{i}^{\beta 2} \alpha_{i}^{\beta 1} \alpha_{i}^{\beta 2}(T)^{2}=1$ due to $T^{2}=1$. Thus $c^{2}=1$ and we obtain

$$
w= \pm v_{i} .
$$


We now start varying $K_{1}^{*}$ and $K_{2}$. Since we are interested in the situation $|\beta|>\beta_{c}$, i.e. $\left|K_{2}\right|>K_{1}^{*}\left(K_{1}^{*}\right.$ is taken to be positive), we change $K_{1}^{*}$ to 0 with fixed $K_{2}$, although a similar argument works for the case $\left|K_{2}\right|<K_{1}^{*}$, in which case we change $K_{2}$ to 0 with $K_{1}^{*}$ fixed. We prove the continuous dependence of $w$ and $v_{i}$ on $K_{1}^{*}$ and $+\operatorname{sign}$ in $(5.35)$ in the limit of $K_{1}^{*}=0$. This would prove

$$
w=v_{i} .
$$

By (3.7), (3.8), and (3.9) of [5], $H(\theta)$ is a continuous function of $e^{i \theta}$ and the parameters $\left(K_{1}^{*}, K_{2}\right)$. Hence, if $\bar{H}(\theta)$ denotes $H(\theta)$ for other values $\left(\bar{K}_{1}^{*}, \bar{K}_{2}\right)$ of these parameters, then $\sup _{\theta}|\bar{H}(\theta)-H(\theta)|$ tends to 0 as $\left(\bar{K}_{1}^{*}, \bar{K}_{2}\right) \rightarrow\left(K_{1}^{*}, K_{2}\right)$ (assuming $|\beta| \neq \beta_{c}$ ). Therefore, the operator $H$ (on the test function space) is continuous in $\left(K_{1}^{*}, K_{2}\right)$ relative to the norm topology. Furthermore, $|\bar{H}(\theta)-H(\theta)|$ tends to 0 as $\left(\bar{K}_{1}^{*}, \bar{K}_{2}\right) \rightarrow\left(K_{1}^{*}, K_{2}\right)$ uniformly over complex values of $z=e^{i \theta}$ in a sufficiently small neighbourhood of the unit circle $|z|=1$. Thus by the same estimate as the proof of Lemma 2 in [7],

$$
\begin{gathered}
(\bar{H}-H)_{k j} \leqq c(1 \pm \varepsilon)^{k-j}, \\
c=\sup _{|z|=1 \pm \varepsilon}|\bar{H}(\theta)-H(\theta)| \rightarrow 0 \quad \text { as } \quad\left(\bar{K}_{1}^{*}, \bar{K}_{2}\right) \rightarrow\left(K_{1}^{*}, K_{2}\right) .
\end{gathered}
$$

By the proof of Lemma 2 of [7], this implies that the operator $\Delta$ of $(5.10)$ is continuous in the parameters $\left(K_{1}^{*}, K_{2}\right)$ relative to the trace class norm. Therefore, $\tilde{\Delta}(z)$ defined by $(5.15)$ is continuous in $\left(K_{1}^{*}, K_{2}\right)$ relative to the norm topology, the continuity being uniform in $z$ over a compact set in the complex plane. By $(5.16), v_{i}$ is continuous in $\left(K_{1}^{*}, K_{2}\right)$ relative to the norm topology.

Next we prove the continuous dependence of $w$ on $\left(K_{1}^{*}, K_{2}\right)$. We use the definition (5.31) and the expression $T=B(h) \sigma_{x}^{(1)}$. By (5.28) and (5.29), $\alpha_{i}^{\beta 2} \alpha_{i}^{\beta 1} \alpha_{i}^{\beta 2}(B(h))$ is entire analytic in $\left(K_{1}^{*}, K_{2}\right)$ relative to the operator norm. On the other hand, Lemma 4.1 implies the entire analyticity of $\alpha_{z}^{\beta 1}(A)$ and $\alpha_{z}^{\beta 2}(A)$ in the parameters $z K_{1}^{*}$ and $z K_{2}$ for any strictly local $A$. Therefore $\alpha_{i}^{\beta 2} \alpha_{i}^{\beta 1} \alpha_{i}^{\beta 2}\left(\sigma_{x}^{(1)}\right)$, and hence $w$ is entire analytic in $\left(K_{1}^{*}, K_{2}\right)$.

Finally, we compute $v_{i}$ and $w$ for $K_{1}^{*}=0$. Then $H=K_{2} H_{2}$ and

$$
\alpha_{t}^{\beta}(A)=\left(\alpha_{t}^{\beta 2}\right)^{2}(A)
$$

for $A \in \mathfrak{A}^{\text {CAR }}$ by (5.29) and (5.3). We have seen that the limit (4.7) exists for $Q$ in a total subset of $\hat{\mathfrak{A}}$, namely for strictly local $Q \in \mathfrak{U}$ in (4.7) and for $Q=B(h)$ in (5.29). Therefore, restricting for $z=t$ real, we obtain the existence of the limit (4.7) for all $Q \in \hat{\mathfrak{U}}$ and it defines a one parameter group of $*$-automorphisms $\alpha_{t}^{\beta 2}$ of $\hat{\mathfrak{A}}$. By Proposition 5.1 (2), we obtain

$$
\hat{\alpha}_{t}^{\beta}=\left(\alpha_{t}^{\beta 2}\right)^{2}
$$

in the present case. As we know the existence of analytic continuation for $\hat{\alpha}_{t}^{\beta}(T)$ and $\alpha_{t}^{\beta 2}(T)$, we obtain

$$
v_{i}=\hat{\alpha}_{i}^{\beta}(T) T=\left(\alpha_{i}^{\beta 2}\right)^{2}(T) T=w .
$$

(In the case of $K_{2}=0, \alpha_{t}^{\beta}$ commutes with $T$ and $v_{i}=w=1$.) Q.E.D. 
Proposition 5.7. $\varphi_{\beta}$ and $\varphi_{\beta \pm}$ are ground states of $\left(\mathfrak{H}, \alpha_{t}^{\beta}\right)$. If $K_{1}^{*} \geqq\left|K_{2}\right|>0, \varphi_{\beta}$ is the unique ground state. If $0<K_{1}^{*}<\left|K_{2}\right|, \varphi_{\beta \pm}$ are the only pure ground states and the cyclic representations of $\mathfrak{U}$ associated with $\varphi_{\beta \pm}$ are mutually disjoint.

Proof. The time translation $\alpha_{t}^{\mathrm{CAR}}$ is a quasifree dynamics of $\mathfrak{A}^{\mathrm{CAR}}$ determined by $\alpha_{t}^{\mathrm{CAR}}(B(h))=B\left(e^{-2 i t H} h\right)$ and the projection operator $E$ described by (3.3) is the spectral projection of the generator $-2 H$ for the interval $(0,+\infty)$. Since $H$ does not have an eigenvalue 0 if $K_{1}^{*}, K_{2} \neq 0$ by Lemma 3.1 of [5], the Fock state $\varphi_{E}$ is the unique ground state of $\left(\mathfrak{Q}^{\mathrm{CAR}}, \alpha_{t}^{\mathrm{CAR}}\right)$ (by Theorem 3 (1) of [6], for example). The restriction of $\varphi_{E}$ to $\mathfrak{Q}_{+}^{\mathrm{CAR}}=\mathfrak{A}_{+}$is the unique ground state of $\left(\mathfrak{A}_{+}, \alpha_{t}^{\beta}\right)$ if $K_{1}^{*} \neq 0$ and $K_{2} \neq 0$ by Theorem 4 (1) of [6]. Note that $H$ has a continuous spectrum if $K_{1}^{*} \neq 0$ and $K_{2} \neq 0$. Hence $\varphi_{\beta}$ is the only $\Theta$-invariant ground state. We can then find all ground states of $\left(\mathfrak{A}, \alpha_{t}^{\beta}\right)$ according to the scheme described in Theorem 5 of [6]. The relevant criterion has been worked out in [5] and the conclusion is that $\varphi_{\beta}$ is the unique ground state of $\left(\mathfrak{A}, \alpha_{t}^{\beta}\right)$ if $K_{1}^{*} \geqq\left|K_{2}\right|>0$ and that $\varphi_{\beta}=\left(\varphi_{\beta+}+\varphi_{\beta-}\right) / 2$ if $0<K_{1}^{*}$ $<\left|K_{2}\right|$, where $\varphi_{\beta \pm}$ are the only pure ground states of $\left(\mathfrak{A}, \alpha_{t}^{\beta}\right)$ and yield mutually disjoint representations of $\mathfrak{A}$. Q.E.D.

Remark 5.8. If $K_{2}=0$, the original system is the same as the tensor product of onedimensional Ising model and a time independent spin-lattice system. The corresponding one-dimensional system $\left(\mathfrak{H}, \alpha_{t}^{\beta}\right)$ has a unique ground state [though $\left(\mathfrak{A}_{+}, \alpha_{t}^{\beta}\right)$ has an infinite number of ground states].

The case $K_{1}^{*}=0$ does not correspond to any finite parameter values of the original two-dimensional system.

\section{Clustering Properties}

Let $\varphi$ be a pure ground state of $\left(\mathfrak{A}, \alpha_{t}\right)$ and $(\mathfrak{H}, \pi, \Phi)$ be the (GNS) triplet of a Hilbert space, a representation of $\mathfrak{U}$ and a cyclic vector for the state $\varphi: \varphi(A)=(\Phi, \pi(A) \Phi)$. Let $U_{t}$ be the canonical one-parameter group of unitaries on $\mathfrak{H}$ implementing $\alpha_{t}$ :

Let $U_{t}=e^{i t L}$.

$$
U_{t} \pi(A) \Phi=\pi\left(\alpha_{t}(A)\right) \Phi .
$$

The following properties of the generator $L$ will be relevant for the general discussion in this section:

(i) $L \geqq 0$.

(ii) The multiplicity of the point spectrum $O$ of $L$ is 1 .

(iii) There is a gap of $\delta>0$ between $O$ and the rest of the spectrum of $L$ [namely $(0, \delta) \cap \operatorname{Spec} L=\emptyset$ and $\delta \in \operatorname{Spec} L]$ and $\delta$ is not a point spectrum of $L$.

First, we discuss the validity of these properties. Then we discuss its consequence. Combining these discussions, we obtain finally our conclusion about clustering properties of $\varphi_{\beta \pm}$ and $\varphi_{\beta}$.

The property (i) is a definition of an $\alpha_{t}$-invariant state $\varphi$ being a ground state. The property (ii) follows from an abstract property of $\varphi$, which is satisfied by $\varphi_{\beta}$ (when $0<\left|K_{2}\right| \leqq K_{1}^{*}$ ) and by $\varphi_{\beta \pm}$ (when $\left|K_{2}\right|>K_{1}^{*}>0$ ) due to Proposition 5.7:

Proposition 6.1. Assume that $\varphi$ is a pure ground state and the associated representation is disjoint from representations associated with any other pure ground states, then (ii) is satisfied. 
Proof. If $\Phi^{\prime}$ is a vector annihilated by $L$, then $\omega^{\prime}(A)=\left(\Phi^{\prime}, \pi(A) \Phi^{\prime}\right)$ is a ground state and the associated (GNS) representation [is contained in $(\pi, \mathfrak{H})$ and hence] must be the same as $(\pi, \mathfrak{H})$ because of the irreducibility of $\pi$. Therefore, $\omega^{\prime}$ is a pure ground state with the associated representation coinciding with that of $\omega$. By assumption $\omega^{\prime}=\omega$. By the irreducibility of $\pi, \Phi^{\prime}=c \Phi$ with a complex number $c$. Q.E.D.

The property (iii) requires a concrete computation.

Proposition 6.2. (1) For $\left|K_{2}\right|>K_{1}^{*}>0$, pure ground states $\varphi_{\beta \pm}$ satisfy (iii) with $\delta=4\left(\left|K_{2}\right|-K_{1}^{*}\right)$.

(2) For $0<\left|K_{2}\right|<K_{1}^{*}$, the pure ground state $\varphi_{\beta}$ satisfies (iii) with $\delta=2\left(K_{1}^{*}-\left|K_{2}\right|\right)$.

Proof. From the formula (5.9) for $H(\theta)$ and the definition (3.8) of [5] for $\gamma(\theta), H$ has an absolutely continuous spectrum of multiplicity 1 on

$$
\begin{aligned}
\operatorname{Spec} H= & {\left[-\left(\left|K_{2}\right|+K_{1}^{*}\right),-|| K_{2}\left|-K_{1}^{*}\right|\right] } \\
& \cup\left[|| K_{2}\left|-K_{1}^{*}\right|,\left|K_{2}\right|+K_{1}^{*}\right] .
\end{aligned}
$$

For the ground state $\varphi_{E}$ of ( $\left.\mathfrak{U}^{\mathrm{CAR}}, \alpha_{t}^{\mathrm{CAR}}\right)$, we may define $L^{\mathrm{CAR}}$ in exactly the same manner as above. Since $\alpha_{t}^{\mathrm{CAR}}(B(h))=B\left(e^{-2 i H t} h\right), L^{\mathrm{CAR}}$ satisfies the property (i), (ii), and (iii) with $\delta=2|| K_{2}\left|-K_{1}^{*}\right|$. Furthermore, the restriction $L_{+}^{\mathrm{CAR}}$ of $L^{\mathrm{CAR}}$ to the even subspace, which is the same as $L$ constructed for the restriction of $\varphi_{E}$ to $\left(\mathfrak{A}_{+}^{\mathrm{CAR}}, \alpha_{t}^{\mathrm{CAR}}\right)=\left(\mathfrak{U}_{+}, \alpha_{t}^{\beta}\right)$, satisfies the property (i), (ii), and (iii) with $\delta=4|| K_{2}\left|-K_{1}^{*}\right|$. (These follow from the formula $U_{t}=\sum_{n=0}^{\infty}\left(e^{-2 i t H}\right)^{\otimes n}$ on a Fock space $\mathfrak{H}=\sum_{n=0}^{\infty} \operatorname{Antisym}\left[(E \mathfrak{R})^{\otimes n}\right], \mathfrak{R}=l_{2}(\mathbb{Z}) \oplus l_{2}(\mathbb{Z})$.)

When $\left|K_{2}\right|>K_{1}^{*}>0$, we can apply Proposition 5.1 (2) of [6] and obtain the GNS representations for $\left(\mathfrak{A}, \alpha_{t}^{\beta}, \varphi_{\beta \pm}\right)$ in the GNS representation space of $\left(\mathfrak{A}_{+}, \alpha_{t}^{\beta}, \varphi_{\beta}\right)=\left(\mathfrak{A}_{+}^{\mathrm{CAR}}, \alpha_{t}^{\mathrm{CAR}}, \varphi_{E}\right)$ with the identical cyclic vector and with the identical representation of $\mathfrak{A}_{+} \subset \mathfrak{A}$. Since $U_{t}$ is determined already by the cyclic vector and the representation of $\mathfrak{A}_{+}$[via (6.1) valid on a dense set $\left.\pi\left(\mathfrak{A}_{+}\right) \Phi\right]$, we obtain $L=L_{+}^{\mathrm{CAR}}$, which satisfies (i), (ii), and (iii) with $\delta=4\left(\left|\mathrm{~K}_{2}\right|-\mathrm{K}_{1}^{*}\right)$.

Now we consider the case $0<\left|K_{2}\right|<K_{1}^{*}$. We imitate Proposition 5.1 of [6] and perform an irreducible decomposition of the cyclic representation $\hat{\pi}$ of $\hat{\mathfrak{A}}$ associated with the state $\hat{\varphi}_{E}$ of $\hat{\mathfrak{A}}$ given by

$$
\hat{\varphi}_{E}\left(A_{1}+T A_{2}\right)=\varphi_{E}\left(A_{1}\right), \quad A_{1}, A_{2} \in \mathfrak{A}^{\mathrm{CAR}} .
$$

Like (4.2) (4.4) of [6], the cyclic representation $(\hat{\pi}, \hat{\mathfrak{H}})$ of $\hat{\mathfrak{A}}$ associated with this state can be decomposed as a sum of 4 irreducible representations $\left(\pi_{i j}, \mathfrak{S}_{i j}\right)$, $i, j=1,2$, of $\mathfrak{A}_{+}^{\mathrm{CAR}}=\mathfrak{A}_{+}$:

$$
\begin{aligned}
\hat{\mathfrak{H}}_{11}=\left(\hat{\pi}\left(\mathfrak{H}_{+}^{\mathrm{CAR}}\right) \hat{\Phi}\right)^{-}, & \hat{\mathfrak{H}}_{12}=\left(\hat{\pi}\left(\mathfrak{H}_{-}^{\mathrm{CAR}}\right) \hat{\Phi}\right)^{-}, \\
\hat{\mathfrak{H}}_{21}=\left(\hat{\pi}\left(\mathfrak{H}_{+}^{\mathrm{CAR}} T\right) \hat{\Phi}\right)^{-}, & \hat{\mathfrak{H}}_{22}=\left(\hat{\pi}\left(\mathfrak{H}_{-}^{\mathrm{CAR}} T\right) \hat{\Phi}\right)^{-},
\end{aligned}
$$

where $\hat{\Phi}$ is the cyclic representative vector for the state $\hat{\varphi}_{E}$. It can be combined into two irreducible Fock representations of $\mathfrak{A}^{\mathrm{CAR}}=\mathfrak{A}_{+}^{\mathrm{CAR}}+\mathfrak{A}_{-}^{\mathrm{CAR}}$ on $\hat{\mathfrak{H}}_{1}=\hat{\mathfrak{H}}_{11}+\hat{\mathfrak{H}}_{12}$ 
and $\hat{\mathfrak{H}}_{2}=\hat{\mathfrak{H}}_{21}+\hat{\mathfrak{H}}_{22}$, associated with Fock states $\varphi_{E}$ and $\varphi_{\theta_{-} E \theta_{-}}$, respectively. By Lemma 5.1 of [5], these two representations are equivalent, i.e. there exists a unitary mapping $w_{12}$ from $\widehat{\mathfrak{H}}_{2}$ onto $\hat{\mathfrak{H}}_{1}$, intertwining $\pi_{j}^{\text {CAR }}(A)=\left.\hat{\pi}(A)\right|_{\hat{\mathfrak{H}}_{j}}$ of $A \in \mathfrak{U}^{\mathrm{CAR}}$, $j=1,2$. The operators $\hat{\pi}(T) w_{12}^{*}$ and $w_{12} \hat{\pi}(T)$ on $\mathfrak{S}_{1}$ both implement the automorphism $\Theta_{-}$of $\mathfrak{U}^{\mathrm{CAR}}$. Since $\left.\hat{\pi}\left(\mathfrak{U}^{\mathrm{CAR}}\right)\right|_{\hat{\mathfrak{S}}_{1}}$ is irreducible, they can differ only by multiplication of a complex number: $\hat{\pi}(T) w_{12}^{*}=e^{i \alpha} w_{12} \hat{\pi}(T)$. By redefining $e^{i \alpha / 2} w_{12}$ as new $w_{12}$, we obtain the equality $\hat{\pi}(T) w_{12}^{*}=w_{12} \hat{\pi}(T)$ on $\hat{\mathfrak{H}}_{1}$.

Defining $W\left(\xi_{1}+\xi_{2}\right)=w_{12}^{*} \xi_{1}+w_{12} \xi_{2}$ for $\xi_{j} \in \hat{\mathfrak{H}}_{j}$, we obtain a selfadjoint unitary operator $W$, which commutes with $\hat{\pi}(A), A \in \mathfrak{A}^{\mathrm{CAR}}$ (by the intertwining property of $w_{12}$ and $w_{12}^{*}$ ) and with $\hat{\pi}(T)\left[\right.$ by $\left.\hat{\pi}(T) w_{12}^{*}=w_{12} \hat{\pi}(T)\right]$. Therefore, $W$ is in $\hat{\pi}(\hat{\mathfrak{H}})^{\prime}$. We have an orthogonal decomposition:

$$
\begin{gathered}
\hat{\mathfrak{H}}=\hat{\mathfrak{H}}_{+} \oplus \hat{\mathfrak{H}}_{-}, \quad \hat{\mathfrak{H}}_{ \pm}=(\mathbf{1} \pm W) \hat{\mathfrak{H}}, \\
\hat{\Phi}=\left(\hat{\Phi}_{+} \oplus \hat{\Phi}_{-}\right) / \sqrt{2}, \quad \hat{\Phi}_{ \pm}=2^{-1 / 2}(\mathbf{1} \pm W) \hat{\Phi}, \\
\hat{\pi}(A)=\hat{\pi}_{+}(A) \oplus \hat{\pi}_{-}(A), \quad A \in \hat{\mathfrak{U}}, \\
\hat{\varphi}_{E}=\left(\hat{\varphi}_{E+}+\hat{\varphi}_{E-}\right) / 2, \quad \hat{\varphi}_{E \pm}(A)=\left(\hat{\Phi}_{ \pm}, \hat{\pi}(A) \hat{\Phi}_{ \pm}\right) .
\end{gathered}
$$

The cyclic representations $\hat{\pi}_{ \pm}$associated with states $\hat{\varphi}_{E \pm}$ of $\hat{\mathfrak{U}}$ can be constructed on $\hat{\mathfrak{H}}_{1}$ by

$$
\hat{\pi}_{ \pm}\left(A_{1}+A_{2} T\right)=\left.\left(\hat{\pi}\left(A_{1}\right) \pm \hat{\pi}\left(A_{2} T\right) W\right)\right|_{\hat{\mathfrak{H}}_{1}}, \quad A_{i} \in \mathfrak{A}^{\mathrm{CAR}},
$$

with the same cyclic vector $\hat{\Phi}:\left(\hat{\Phi}, \hat{\pi}_{ \pm}(A) \hat{\Phi}\right)=\hat{\varphi}_{E \pm}(A), A \in \hat{\mathfrak{A}}$. Each $\hat{\pi}_{ \pm}$is irreducible as $\hat{\pi}_{ \pm}\left(\mathfrak{U}^{\mathrm{CAR}}\right)=\left.\hat{\pi}\left(\mathfrak{A}^{\mathrm{CAR}}\right)\right|_{\hat{\mathfrak{H}}_{1}}$ is already irreducible.

Since $\left.\hat{\pi}\left(\mathfrak{U}^{\mathrm{CAR}}\right)\right|_{\hat{\mathfrak{H}}_{1}}$ is irreducible, there exists a net $A_{v} \in \mathfrak{U}^{\mathrm{CAR}}$ such that $\hat{\pi}\left(A_{v}\right)$ $\rightarrow \hat{\pi}(T) w_{12}^{*}=w_{12} \hat{\pi}(T)$ on $\hat{\mathfrak{H}}_{1}$. Since $w_{12}$ intertwine $\hat{\pi}\left(\mathfrak{H}^{\mathrm{CAR}}\right)$ on $H_{2}$ and $H_{1}$, we obtain $\hat{\pi}\left(A_{v}\right) \rightarrow \hat{\pi}(T) W\left[=\hat{\pi}(T) w_{12}^{*} \oplus \hat{\pi}(T) w_{12}\right.$ on $\left.\hat{H}_{1} \oplus \hat{H}_{2}\right]$. This means $\hat{\pi}_{ \pm}\left(A_{v} T\right)$ $\rightarrow \pm(\hat{\pi}(T) W)^{2}= \pm 1$ and hence $\hat{\pi}_{ \pm}$are mutually disjoint irreducible representations. Since $\varphi_{E}$ is $\hat{\alpha}_{t}$ invariant by (6.3) due to the $\alpha_{t}^{\mathrm{CAR}}$-invariance of $\varphi_{E}$, the decomposition (6.8) into disjoint pure states implies the $\alpha_{t}$-invariance of each of $\hat{\varphi}_{E \pm}$. The associated operator $U_{t}$ for $\left(\hat{\mathfrak{U}}, \hat{\alpha}_{t}, \hat{\varphi}_{E \pm}\right)$ is already determined on the dense set $\hat{\pi}\left(\mathfrak{A}^{\mathrm{CAR}}\right) \hat{\Phi}=\pi\left(\mathfrak{A}^{\mathrm{CAR}}\right) \Phi$ in $\hat{\mathfrak{S}}_{1}$ and hence its generator $\hat{L}$ coincides with $L^{\mathrm{CAR}}$ associated with the Fock state $\varphi_{E}$ of $\mathfrak{A}^{\mathrm{CAR}}$. It then satisfies (i), (ii), and (iii) with $\delta=2\left(K_{1}^{*}-\left|K_{2}\right|\right)$.

We now restrict $\hat{\pi}_{ \pm}$to $\mathfrak{A} \subset \hat{\mathfrak{U}}$. We are considering Case (A) in Sect. 6 of [5]. Therefore, $w_{12}$ maps the even part $\hat{\mathfrak{H}}_{11}$ of $\hat{\pi}\left(\mathfrak{U}^{\mathrm{CAR}}\right) \hat{\mathfrak{H}}_{1}$ onto the even part $\hat{\mathfrak{H}}_{21}$ of $\hat{\pi}\left(\mathfrak{U}^{\mathrm{CAR}}\right) \hat{\mathfrak{H}}_{2}$ and $\hat{\pi}(T) W \in \hat{\pi}\left(\mathfrak{U}_{+}^{\mathrm{CAR}}\right)^{\prime \prime}$. Consequently, $\hat{\varphi}_{E \pm}(A T)= \pm(\Phi, \hat{\pi}(A) \hat{\pi}(T) W \Phi)$ $=0$ for $A \in \mathfrak{U}_{-}^{\mathrm{CAR}}$, and we obtain

$$
\varphi^{\beta}(A)=\hat{\varphi}_{E \pm}(A), \quad A \in \mathfrak{U} .
$$

Since $\hat{\mathfrak{H}}_{11}$ and $\hat{\mathfrak{H}}_{12}$ yield mutually non-equivalent irreducible representations of $\mathfrak{U}_{+}^{\mathrm{CAR}}=\mathfrak{A}_{+}$due to Lemma 4.1 (1) of [6], for example, and since $\hat{\pi}_{ \pm}(A T)$ $= \pm \hat{\pi}(A)(\hat{\pi}(T) W)$ bridges $\hat{\mathfrak{H}}_{11}$ and $\hat{\mathfrak{H}}_{12}$ for $A \in \mathfrak{U}_{-}^{\text {CAR }}$ [due to $\left.\hat{\pi}(T) W \in \hat{\pi}\left(\mathfrak{U}_{+}^{\text {CAR }}\right)^{\prime \prime}\right]$, $\hat{\Phi}$ is cyclic for $\hat{\pi}_{ \pm}(\mathfrak{U})=\hat{\pi}_{ \pm}\left(\mathfrak{U}_{+}^{\mathrm{CAR}}\right)+\hat{\pi}_{ \pm}\left(\mathfrak{U}_{-}^{\mathrm{CAR}} T\right)$. Therefore, $\left(\hat{\mathfrak{H}}_{1}, \hat{\pi}_{+}, \hat{\Phi}\right)$ provides the GNS representation for $\varphi^{\beta}$ and the $U_{t}$ for $\varphi^{\beta}$ (in this concrete representation) coincides with the $U_{t}$ for $\left(\hat{\mathfrak{A}}, \hat{\alpha}_{t}, \hat{\varphi}_{E \pm}\right)$ discussed above. Hence $L$ in this case satisfies (i), (ii), and (iii) with $\delta=2\left(K_{1}^{*}-\left|K_{2}\right|\right)$. Q.E.D. 
We now consider a state $\psi$ on the two-dimensional system which is related to $\varphi$ by

$$
\begin{aligned}
& \psi\left(\xi\left(k_{1}, I_{1}\right) \ldots \xi\left(k_{n}, I_{n}\right)\right) \\
& \quad=\left(\Phi, \pi\left(\sigma_{x}\left(I_{1}\right)\right) e^{-\left(k_{2}-k_{1}\right) L} \pi\left(\sigma_{x}\left(I_{2}\right)\right) \ldots e^{-\left(k_{n}-k_{n-1}\right) L} \pi\left(\sigma_{x}\left(I_{n}\right)\right) \Phi\right)
\end{aligned}
$$

for $k_{1}<k_{2}<\ldots<k_{n}$. We note that

$$
\begin{aligned}
& \varphi\left(\alpha_{t_{1}}\left(A_{1}\right) \ldots \alpha_{t_{n}}\left(A_{n}\right)\right) \\
& \quad=\left(\Phi, \pi\left(\dot{A}_{1}\right) e^{i\left(t_{2}-t_{1}\right) L} \pi\left(A_{2}\right) \ldots e^{i\left(t_{n}-t_{n-1}\right) L} \pi\left(A_{n}\right) \Phi\right)
\end{aligned}
$$

by (6.1). By $L \geqq 0, e^{i z L}$ is continuous for $\operatorname{Im} z \geqq 0$ and holomorphic for $\operatorname{Im} z>0$ with $\left\|e^{i z L}\right\|=1$ for $\operatorname{Im} z \geqq 0$. Hence the right-hand side of (6.12) defines a bounded continuous function of $\left(t_{1}, \ldots, t_{n}\right) \in \overline{\mathfrak{I}}_{n}$ in the tube domain

$$
\overline{\mathfrak{I}}_{n} \equiv\left\{\left(t_{1}, \ldots, t_{n}\right) \in \mathbb{C}^{n} ; \operatorname{Im}\left(t_{j}-t_{j-1}\right) \geqq 0, j=2, \ldots, n\right\}
$$

satisfying two conditions: (1) it is holomorphic for $\left(z_{1}, \ldots, z_{n}\right) \in \mathfrak{J}_{n} \equiv$ the interior of $\overline{\mathfrak{I}}_{n}$ and $(2)$ it coincides with $\varphi\left(\alpha_{t_{1}}\left(A_{1}\right) \ldots \alpha_{t_{n}}\left(A_{n}\right)\right)$ for real values of $t$ 's. The right-hand side of $(6.11)$ is then the value of this function for $t_{j}=i k_{j}$ and is called the (n-point) Schwinger function of $\varphi$.

Let us recall that $\tau_{(l, m)}$ is the lattive translation automorphism of the twodimensional system:

$$
\tau_{(l, m)}(\xi(k, I))=\xi(k+l, I+m),
$$

where $I+m=\{j+m ; j \in I\}$, and $\tau_{n}$ is the lattice translation of the one-dimensional system:

$$
\tau_{n}\left(\sigma_{\alpha}^{(j)}\right)=\sigma_{\alpha}^{(j+n)}
$$

We now discuss consequences of the properties (ii) and (iii) for $L$ separately.

Proposition 6.3. If $\varphi$ is a pure ground state of $\left(\mathfrak{A}, \alpha_{t}\right)$ and L satisfies (ii), then the state $\psi$ related to $\varphi$ by (6.11) is invariant under the translations $(l, 0), l \in \mathbb{Z}$, and satisfies

$$
\lim _{l \rightarrow \infty} \psi\left(F_{1} \tau_{(l, 0)}\left(F_{2}\right)\right)=\psi\left(F_{1}\right) \psi\left(F_{2}\right)
$$

for any elements $F_{1}$ and $F_{2}$ in the $C^{*}$-algebra 0 generated by $\xi$ 's.

Proof. The invariance is a direct consequence of (6.11). Let

$$
F_{1}=\prod_{i=1}^{n_{1}} \xi\left(k_{i 1}, I_{i 1}\right), \quad F_{2}=\prod_{i=1}^{n_{2}} \xi\left(k_{i 2}, I_{i 2}\right)
$$

with $k_{1 j}<k_{2 j}<\ldots<k_{n_{j} j}$ for $j=1,2$. For sufficiently large $l>0$, we have $l^{\prime} \equiv k_{12}+l$ $-k_{n_{1} 1}>0$ and by $(6.11)$

$$
\begin{gathered}
\psi\left(F_{1} \tau_{(i, 0)}\left(F_{2}\right)\right)=\left(\Phi_{1}, e^{-l^{\prime} L} \Phi_{2}\right), \\
\Phi_{1}=\pi\left(\sigma_{x}\left(I_{n_{1} 1}\right)\right) e^{-\left(k_{n_{1} 1}-k_{n_{1}}-1,1\right) L} \ldots \pi\left(\sigma_{x}\left(I_{11}\right)\right) \Phi \\
\Phi_{2}=\pi\left(\sigma_{x}\left(I_{12}\right)\right) e^{-\left(k_{22}-k_{12}\right) L} \ldots \pi\left(\sigma_{x}\left(I_{n_{2}}\right)\right) \Phi
\end{gathered}
$$


Since $\lim _{l^{\prime} \rightarrow+\infty} e^{-l^{\prime} L}=$ the spectral projection $E_{L}(0)$ for the point spectrum $O$ of $L$, which is the one-dimensional projection on $\Phi$ by the assumed property (ii) for $L$, we obtain

$$
\lim _{l \rightarrow+\infty} \psi\left(F_{1} \tau_{(l, 0)}\left(F_{2}\right)\right)=\left(\Phi_{1}, \Phi\right)\left(\Phi, \Phi_{2}\right)=\psi\left(F_{1}\right) \psi\left(F_{2}\right) .
$$

The same argument works for $l \rightarrow-\infty$. By linear combination and approximation in norm, we obtain (6.14) for a general $F_{1}$ and $F_{2}$. Q.E.D.

Corollary 6.4. If $\left[\alpha_{t}, \tau_{n}\right]=0, \varphi \circ \tau_{n}=\varphi$ and L satisfies (ii), then $\psi$ is invariant under $\tau_{(l, m)}$ and satisfies

$$
\lim _{(l, m) \rightarrow \infty} \psi\left(F_{1} \tau_{(l, m)}\left(F_{2}\right)\right)=\psi\left(F_{1}\right) \psi\left(F_{2}\right) .
$$

Proof. By the translational invariance of $\varphi$, there exists a unitary operator $U$ such that

$$
U^{n} \pi(A) \Phi=\pi\left(\tau_{n}(A)\right) \Phi .
$$

By $\left[\alpha_{t}, \tau_{n}\right]$ and $(6.1)$, we obtain $\left[e^{i L t}, U\right]=0$. Thus the invariance $\psi=\psi \tau_{(l, m)}$ is immediate from (6.11):

$$
\begin{aligned}
\psi( & \left.\left.\tau_{(l, m)}\right)\left(\xi\left(k_{1}, I_{1}\right) \ldots \xi\left(k_{n}, I_{n}\right)\right)\right) \\
& =\psi\left(\xi\left(k_{1}+l, I_{1}+m\right) \ldots \xi\left(k_{n}+l, I_{n}+m\right)\right) \\
& =\left(\Phi, U^{m} \pi\left(\sigma_{x}\left(I_{1}\right)\right) U^{-m} e^{-\left(k_{2}-k_{1}\right) L} \ldots e^{-\left(k_{n}-k_{n-1}\right) L} U^{m} \pi\left(\sigma_{x}\left(I_{n}\right)\right) U^{-m} \Phi\right) \\
& =\left(\Phi, \pi\left(\sigma_{x}\left(I_{1}\right)\right) e^{-\left(k_{2}-k_{1}\right) L} \ldots e^{-\left(k_{n}-k_{n-1}\right) L} \pi\left(\sigma_{x}\left(I_{n}\right)\right) \Phi\right) \\
& =\psi\left(\xi\left(k_{1}, I_{1}\right) \ldots \xi\left(k_{n}, I_{n}\right)\right),
\end{aligned}
$$

where the third equality is due to $U^{-m} \Phi=\Phi$ and $U^{-m} e^{-k L} U^{m}=e^{-k L}$.

Given $\varepsilon>0$, there exists a $\Delta>0$ such that

$$
\left\|\left\{E_{L}([0, \Delta])-E_{L}(0)\right\} \Phi_{j}\right\|<\varepsilon^{1 / 2} \quad(j=1,2),
$$

where $\Phi_{1}$ and $\Phi_{2}$ are given by $(6.17)$ and $(6.18)$, and $E_{L}([0, \Delta])$ is the spectral projection of $L$ for the interval $[0, \Delta]$. For any $l>N_{+}$with a natural number $N_{+}$

$$
N_{+} \geqq \Delta^{-1} \log \left(\left\|\Phi_{1}\right\|\left\|\Phi_{2}^{\prime}\right\| / \varepsilon\right)+k_{n_{1} 1}-k_{12},
$$

we obtain

$$
\begin{aligned}
\mid \psi( & \left.F_{1}, \tau_{(l, m)}\left(F_{2}\right)\right)-\psi\left(F_{1}\right) \psi\left(F_{2}\right) \mid \\
= & \mid\left(\Phi_{1}, E_{L}((\Delta, \infty)) e^{-\left(l+k_{12}-k_{n_{1} 1}\right) L} U^{m} \Phi_{2}\right) \\
& +\left(\left\{E_{L}([0, \Delta])-E_{L}(0)\right\} \Phi_{1}, e^{-\left(l+k_{12}-k_{n_{1} 1}\right) L} U^{m}\left\{E_{L}([0, \Delta])-E_{L}(0)\right\} \Phi_{2}\right) \mid \\
\leqq & \left\|\Phi_{1}\right\|\left\|\Phi_{2}\right\| e^{-\left(l+k_{12}-k_{n_{1}}\right) \Delta}+\prod_{j=1}^{2}\left\|\left\{E_{L}([0, \Delta])-E_{L}(0)\right\} \Phi_{j}\right\|<2 \varepsilon .
\end{aligned}
$$

A similar estimate holds for any $l<-N_{-}$with a suitable natural number $N_{-}$. For each $l \in\left[-N_{-}, N_{+}\right]$, we obtain

$$
\begin{aligned}
\lim _{m \rightarrow \infty} \psi\left(F_{1} \tau_{(l, m)}\left(F_{2}\right)\right) & =\psi\left(F_{1}\right) \psi\left(\tau_{(l, 0)}\left(F_{2}\right)\right) \\
& =\psi\left(F_{1}\right) \psi\left(F_{2}\right)
\end{aligned}
$$


due to (2.3) for $\varphi_{\beta \pm}$ and due to (2.3) for $\varphi_{\beta}$ when $\varphi_{\beta}$ is pure. Therefore, we obtain (6.20) for $F_{1}, F_{2}$ of the form (6.15). By linear combination and approximation in norm, we obtain (6.20) for any $F_{1}$ and $F_{2}$. Q.E.D.

Proposition 6.5. Assume (iii) for $L$.

(1) If $F_{1}$ is any function of $\xi_{(l, m)}$ 's with $l \leqq N$ and $F_{2}$ is any function of $\xi_{(l, m)}$ 's with $l \geqq N+d$, then

$$
\left|\psi\left(F_{1} F_{2}\right)-\psi\left(F_{1}\right) \psi\left(F_{2}\right)\right| \leqq e^{-\delta d} \psi\left(\left|F_{1}\right|^{2}\right)^{1 / 2} \psi\left(\left|F_{2}\right|^{2}\right)^{1 / 2} .
$$

(2) For any polynomials $F_{1}$ and $F_{2}$ of $\xi$ 's

$$
\lim _{l \rightarrow \infty} e^{|l| \delta}\left|\psi\left(F_{1} \tau_{(l, 0)}\left(F_{2}\right)\right)-\psi\left(F_{1}\right) \psi\left(F_{2}\right)\right|=0 .
$$

(3) There exist polynomials $F_{1}$ and $F_{2}$ of $\xi$ 's for any given $\varepsilon>0$ such that

$$
\lim _{l \rightarrow+\infty} e^{l(\delta+\varepsilon)}\left|\psi\left(F_{1}, \tau_{(l, 0)}\left(F_{2}\right)\right)-\psi\left(F_{1}\right) \psi\left(F_{2}\right)\right|=\infty .
$$

A similar statement holds for $l \rightarrow-\infty$.

Proof. (1) It is enough to prove (6.26) for a dense set of $F$ 's given by

$$
F_{j}=\sum_{i=1}^{n_{j}} c_{i j} \prod_{i^{\prime}=1}^{n(i, j)} \xi\left(k_{i i^{\prime} j}, I_{i i^{\prime} j}\right), \quad j=1,2,
$$

with $k_{i n(i, 1) 1}<\ldots<k_{i 11} \leqq N$ and $k_{i n(i, 2) 2}>\ldots>k_{i 12} \geqq N+d$. We obtain

$$
\begin{gathered}
\left|\psi\left(F_{1} F_{2}\right)-\psi\left(F_{1}\right) \psi\left(F_{2}\right)\right|=\left|\left(\Phi_{1},\left(\mathbf{1}-E_{L}(0)\right) e^{-d L} \Phi_{2}\right)\right| \\
\leqq e^{-d \delta}\left\|\Phi_{1}\right\|\left\|\Phi_{2}\right\|
\end{gathered}
$$

due to $L \geqq \delta$ on $\left(\mathbf{1}-E_{L}(0)\right) \mathfrak{H}$, where

$$
\Phi_{j}=\sum_{i=1}^{n_{j}} c_{i j} e^{-a_{i j} L} \pi\left(\sigma_{x}\left(I_{i 1 j}\right)\right) e^{-\varepsilon_{j}\left(k_{i 2 j}-k_{i 1}\right) L} \pi\left(\sigma_{x}\left(I_{i 2 j}\right)\right) \ldots \Phi,
$$

$j=1,2, a_{i 1}=N-k_{i 11}, a_{i 2}=k_{i 12}-N-d, \varepsilon_{1}=-1, \varepsilon_{2}=1$.

In order to compute $\left\|\Phi_{j}\right\|$, we introduce a conjugate linear automorphism $F \rightarrow F^{\dagger}$ of the $C^{*}$-algebra $\mathcal{C}$ generated by $\xi$ 's by

$$
\left(c \prod_{j=1}^{n} \xi\left(k_{j}, I_{j}\right)\right)^{\dagger}=\bar{c} \prod_{j=1}^{n} \xi\left(-k_{j}, I_{j}\right) .
$$

For $F=\prod_{j=1}^{n} \xi\left(k_{j}, I_{j}\right), k_{1}<k_{2}<\ldots<k_{n}$, we obtain

$$
\begin{aligned}
\psi\left(\mathrm{F}^{\dagger}\right) & =\left(\Phi, \pi\left(\sigma\left(I_{n}\right)\right) e^{-\left(k_{n}-k_{n-1}\right) L} \ldots e^{-\left(k_{2}-k_{1}\right) L} \pi\left(\sigma\left(I_{1}\right)\right) \Phi\right) \\
& =\left(\Phi, \pi\left(\sigma\left(I_{1}\right)\right) e^{-\left(k_{2}-k_{1}\right) L} \ldots e^{-\left(k_{n}-k_{n-1}\right) L} \pi\left(\sigma\left(I_{n}\right)\right) \Phi\right)^{*} \\
& =\psi(F)^{*} .
\end{aligned}
$$

This equality extends to all $F$ in $\mathbb{C}$ by conjugate linearity and approximation. [We also have the reflection positivity $\psi\left(F^{\dagger} F\right) \geqq 0$ for any function $F$ of $\xi_{(l, m)}$ 's with $l \geqq 0$.] 
We now obtain

$$
\begin{aligned}
\left\|\Phi_{1}\right\|^{2}= & \psi\left(\tau_{(-N, 0)}\left(F_{1}\right)\left\{\tau_{(-N, 0)}\left(F_{1}\right)\right\}^{\dagger}\right) \\
& \leqq \psi\left(\left|\tau_{(-N, 0)}\left(F_{1}\right)\right|^{2}\right)^{1 / 2} \psi\left(\left|\tau_{(-N, 0)}\left(F_{1}\right)^{\dagger}\right|^{2}\right)^{1 / 2} \\
\left\|\Phi_{2}\right\|^{2}= & \psi\left(\left\{\tau_{(-N-d, 0)}\left(F_{2}\right)\right\}^{\dagger} \tau_{(-N-d, 0)}\left(F_{2}\right)\right) \\
& \leqq \psi\left(\left|\tau_{(-N-d, 0)}\left(F_{2}\right)^{\dagger}\right|^{2}\right)^{1 / 2} \psi\left(\left|\tau_{(-N-d, 0)}\left(F_{2}\right)\right|^{2}\right)^{1 / 2}
\end{aligned}
$$

where Cauchy-Schwarz inequality is used. Because

$$
\begin{gathered}
\psi\left(\left|\tau_{(-N, 0)}\left(F_{j}\right)\right|^{2}\right)=\psi\left(\tau_{(-N, 0)}\left(\left|F_{j}\right|^{2}\right)\right)=\psi\left(\left|F_{j}\right|^{2}\right), \\
\psi\left(\left|\tau_{(-N, 0)}\left(F_{j}\right)^{\dagger}\right|^{2}\right)=\psi\left(\tau_{(-N, 0)}\left(\left|F_{j}\right|^{2}\right)^{\dagger}\right)=\psi\left(\left|F_{j}\right|^{2}\right)
\end{gathered}
$$

due to the translation invariance of $\psi$ and (6.33), we obtain

$$
\left\|\Phi_{j}\right\|^{2} \leqq \psi\left(\left|F_{j}\right|^{2}\right), \quad j=1,2 .
$$

Combining (6.30) and (6.36), we obtain (6.26).

(2) For $l>N$, we have

$$
\begin{aligned}
& e^{l \delta}\left(\psi\left(F_{1} \tau_{(l, 0)}\left(F_{2}\right)\right)-\psi\left(F_{1}\right) \psi\left(F_{2}\right)\right) \\
& \quad=\left(\Phi_{1},\left(1-E_{L}(0)\right) e^{-(l-N)(L-\delta)} \Phi_{2}\right) e^{N \delta}
\end{aligned}
$$

with $\Phi_{1}$ and $\Phi_{2}$ given by (6.31), $N=N_{1}-N_{2}, N_{1}=\max _{i} k_{i 11}, N_{2}=\min _{i} k_{i 12}$, $a_{i 1}=N_{1}-k_{i 11}$, and $a_{i 2}=k_{i 12}-N_{2}$. Since $\Phi_{1}$ and $\Phi_{2}$ are fixed vectors, and $(L-\delta)$ has a positive spectrum without a point spectrum at $O$ when restricted to $\left(1-E_{L}(0)\right) \mathfrak{H}$, we have $\lim _{l \rightarrow+\infty}\left(1-E_{L}(0)\right) e^{-(l-N)(L-\delta)}=0$ and we obtain $(6.27)$ for $l \rightarrow+\infty$. The same argument works for $l \rightarrow-\infty$.

(3) By Lemma 7.5 of the next section, there exists $F=\prod_{i=1}^{n} \xi\left(k_{i}, I_{i}\right), 0 \leqq k_{1}<k_{2}$ $<\ldots<k_{n}$, such that

$$
\begin{gathered}
E_{L}((\delta, \delta+(\varepsilon / 2))) \Phi_{F} \neq 0, \\
\Phi_{F}=e^{-k_{1} L} \pi\left(\sigma_{x}\left(I_{1}\right)\right) e^{-\left(k_{2}-k_{1}\right) L} \ldots \pi\left(\sigma_{x}\left(I_{n}\right)\right) \Phi .
\end{gathered}
$$

For $l \geqq 0$,

$$
\begin{aligned}
\psi\left(F^{\dagger} \tau_{(l, 0)}(F)\right)= & \left(\Phi_{F}, e^{-l L} \Phi_{F}\right) \\
& \geqq\left(\Phi_{F}, e^{-l L} E_{L}((\delta, \delta+(\varepsilon / 2))) \Phi_{F}\right) \\
& \geqq e^{-l(\delta+\varepsilon / 2)}\left\|E_{L}((\delta, \delta+(\varepsilon / 2))) \Phi_{F}\right\|^{2}
\end{aligned}
$$

Hence

$$
\lim _{l \rightarrow+\infty} e^{l(\delta+\varepsilon)} \psi\left(F^{\dagger} \tau_{(l, 0)}(F)\right)=+\infty
$$

A similar estimate holds in the case of $l \rightarrow-\infty$ for

$$
\psi\left(F \tau_{(l, 0)}\left(F^{\dagger}\right)\right)=\psi\left(F^{\dagger} \tau_{(-l, 0)}(F)\right) \text {. Q.E.D. }
$$

Specializing to the case of $\varphi_{\beta \pm}$ and $\varphi_{\beta}$, we have Theorem 2 . 


\section{The Unique Correspondence}

The main purpose of this section is to prove the following where $\mathfrak{C}$ is the $C^{*}$ algebra generated by $\xi$ 's.

Proposition 7.1. If a state $\psi=\psi_{j}$ of $\mathfrak{C}$ is related to a ground state $\varphi_{j}$ of $\mathfrak{A}$ by (6.11) for $j=1,2$, with $\alpha_{t}=\alpha_{t}^{\beta}$, then $\psi_{1}=\psi_{2}$ implies $\varphi_{1}=\varphi_{2}$ so that $\varphi_{1} \neq \varphi_{2}$ implies $\psi_{1} \neq \psi_{2}$.

Since we know $\varphi_{\beta+} \neq \varphi_{\beta-}$ (for $\left|K_{2}\right|>K_{1}^{*}>0$ ), we immediately obtain $\omega_{\beta+} \neq \omega_{\beta-}$ by this proposition. As a preparation, we prove the following lemma, in which we use the fact that $\alpha_{z}\left(\sigma_{x}(I)\right)$ is an entire function of $z$ as well as its explicit form.

Lemma 7.2. In the situation of Proposition 7.1, $\psi_{1}=\psi_{2}$ implies

$$
\varphi_{1}\left(\alpha_{z_{1}}\left(\sigma_{x}\left(I_{1}\right)\right) \ldots \alpha_{z_{n}}\left(\sigma_{x}\left(I_{n}\right)\right)\right)=\varphi_{2}\left(\alpha_{z_{1}}\left(\sigma_{x}\left(I_{1}\right)\right) \ldots \alpha_{z_{n}}\left(\sigma_{x}\left(I_{n}\right)\right)\right)
$$

for all $I_{1} \ldots I_{n}$ and complex $z_{1}, \ldots, z_{n}$.

Proof. We consider the following functions of $z=\left(z_{1}, \ldots, z_{n-1}\right)$ :

$$
F_{j}(z)=\left(\Phi_{j}, \pi_{j}\left(\sigma_{x}\left(I_{1}\right)\right) e^{i z_{1} L_{j}} \pi_{j}\left(\sigma_{x}\left(I_{2}\right)\right) \ldots e^{i z_{n-1} L_{j}} \pi_{j}\left(\sigma_{x}\left(I_{n}\right)\right) \Phi_{j}\right),
$$

where $\left(\mathfrak{H}_{j}, \pi_{j}, \Phi_{j}\right)$ is the GNS triplet associated with $\varphi_{j}$, and $e^{i t L_{j}} \pi_{j}(A) \Phi_{j}$ $=\pi_{j}\left(\alpha_{t}(A)\right) \Phi_{j}, A \in \mathfrak{A}, j=1,2$. Since $\varphi_{j}$ is a ground state, $F_{j}(z)$ is a bounded continuous function of $z$ for $\operatorname{Im} z_{j} \geqq 0, j=1, \ldots$ and, for any subset $I$ of $(1, \ldots, n-1)$, $F_{j}(z)$ is holomorphic in $z_{j}, j \in I$ if $\operatorname{Im} z_{j}>0$ for $j \in I$ and $\operatorname{Im} z_{j} \geqq 0$ for all $j \in I$.

For any real $t$ 's we have

$$
\varphi_{j}\left(\alpha_{t_{1}}\left(\sigma_{x}\left(I_{1}\right)\right) \ldots \alpha_{t_{n}}\left(\sigma_{x}\left(I_{n}\right)\right)\right)=F_{j}\left(t_{2}-t_{1}, \ldots, t_{n}-t_{n-1}\right) .
$$

In view of entire analyticity of $\alpha_{t}\left(\sigma_{x}(I)\right)$, we have

$$
\varphi_{j}\left(\alpha_{z_{1}}\left(\sigma_{x}\left(I_{1}\right)\right) \ldots \alpha_{z_{n}}\left(\sigma_{x}\left(I_{n}\right)\right)\right)=F_{j}\left(z_{2}-z_{1}, \ldots, z_{n}-z_{n-1}\right)
$$

whenever $\operatorname{Im}\left(z_{j}-z_{j-1}\right) \geqq 0, j=2, \ldots, n$. On the other hand,

$$
\psi_{j}\left(\prod_{j=1}^{n} \xi\left(k_{j}, I_{j}\right)\right)=F_{j}\left(i\left(k_{2}-k_{1}\right), \ldots, i\left(k_{n}-k_{n-1}\right)\right)
$$

for any integers $k_{1}<k_{2}<\ldots<k_{n}$. Therefore, $\psi_{1}=\psi_{2}$ implies that $F(z)=F_{1}(z)$ $-F_{2}(z)$ is a bounded continuous function of $z$ for $\operatorname{Im} z_{j} \geqq 0$, holomorphic for $\operatorname{Im} z_{j} \geqq 0$ and vanishing at $z_{j}=i n_{j}, n_{j} \in \mathbb{N}$.

We now use the following corollary of Carlson's theorem [8].

Lemma 7.3. If $f(z)$ is a bounded continuous function of a complex variable $z$ in the upper half plane $\operatorname{Im} z \geqq 0$, holomorphic for $\operatorname{Im} z>0$, and if $f(i k)=0$ for $k=1,2, \ldots$, then $f(z)=0$ for all $z$.

By applying Lemma 7.3 to $f_{1}(z)=F\left(z, i k_{2}, \ldots, i k_{n-1}\right)$, we obtain $F\left(z_{1}, i k_{2}, \ldots, i k_{n-1}\right)=0$ for all $z_{1}$ with $\operatorname{Im} z_{1} \geqq 0$. Recursively, if we obtain 
$F\left(z_{1}, \ldots, z_{l-1}, i k_{l}, \ldots\right)=0$ for $\operatorname{Im} z_{j} \geqq 0, j=1, \ldots, l-1$ and for natural numbers $k_{l}, \ldots$, we apply Lemma 7.3 to $f_{l}(z)=F\left(z_{1}, \ldots, z_{l-1}, z, i k_{l+1}, \ldots\right)$ and obtain $f_{l}(z)=0$ for $\operatorname{Im} z_{j} \geqq 0, j=1, \ldots, l$ and for natural numbers $k_{l+1}, \ldots$. By mathematical induction, we obtain $F(z)=0$ for $\operatorname{Im} z_{j} \geqq 0$. Therefore, we obtain (7.1) in view of (7.4). Q.E.D.

Proposition 7.1 follows from Lemma 7.2 if we prove the following.

Lemma 7.4. $\left\{\alpha_{i k}\left(\sigma_{x}^{(j)}\right) ; k \in \mathbb{Z}, j \in \mathbb{Z}\right\}$ generates $\mathfrak{A}$.

Proof. Let $\mathfrak{A}_{0}$ be the $C^{*}$ subalgebra of $\mathfrak{A}$ generated by $\alpha_{i k}\left(\sigma_{x}^{(j)}\right)$. Since $\sigma_{x}^{(j)}=\alpha_{0}\left(\sigma_{x}^{(j)}\right) \in \mathfrak{A}_{0}, \alpha_{z}^{\beta M 2}(A) \in \mathfrak{A}_{0}$ if $A \in \mathfrak{A}_{0}$, where $\alpha_{z}^{\beta M 2}$ is defined by (4.2). Since $\alpha_{i}\left(\sigma_{x}^{(j)}\right)=\alpha_{i}^{\beta 2} \alpha_{i}^{\beta 1} \alpha_{i}^{\beta 2}\left(\sigma_{x}^{(j)}\right)$ by (5.27) and since it belongs to $\mathfrak{A}_{0}$ and is strictly local by Lemma 4.1, we obtain

$$
\alpha_{i}^{\beta 1} \alpha_{i}^{\beta 2}\left(\sigma_{x}^{(j)}\right)=\alpha_{-i}^{\beta 2} \alpha_{i}\left(\sigma_{x}^{(j)}\right)=\lim _{M \rightarrow \infty} \alpha_{-i}^{\beta M 2}\left(\alpha_{i}\left(\sigma_{x}^{(j)}\right)\right) \in \mathfrak{A}_{0} .
$$

Since $\sigma_{z}^{\beta M 2}\left(\sigma_{x}^{(j)}\right)=\sigma_{x}^{(j)}$, we obtain $\sigma_{i}^{\beta 1}\left(\sigma_{x}^{(j)}\right) \in \mathfrak{A}_{0}$. Since $\sigma_{i}^{\beta 1}\left(\sigma_{x}^{(j)}\right)=\operatorname{Ad}\left(e^{K_{1}^{*} \sigma_{z}^{(j)}}\right)\left(\sigma_{x}^{(j)}\right)$ $=e^{2 K_{1}^{*} \sigma_{z}^{(j)}} \sigma_{x}^{(j)}$, we obtain

$$
e^{2 K_{1}^{*} \sigma_{z}^{(j)}}=\sigma_{i}^{\beta 1}\left(\sigma_{x}^{(j)}\right) \sigma_{x}^{(j)} \in \mathfrak{A}_{0} .
$$

Since $e^{2 K_{1}^{*} \sigma_{z}^{(j)}}=\left(\cosh 2 K_{1}^{*}\right)+\left(\sinh 2 K_{1}^{*}\right) \sigma_{z}^{(j)}$ and $\sinh 2 K_{1}^{*} \neq 0$ for $K_{1}^{*} \neq 0$, we obtain $\sigma_{z}^{(j)} \in \mathfrak{A}_{0}$. Hence $\sigma_{y}^{(j)}=i \sigma_{x}^{(j)} \sigma_{z}^{(j)} \in \mathfrak{A}_{0}$ and we have $\mathfrak{A}_{0}=\mathfrak{A}$. Q.E.D.

By a similar method, we obtain the following lemma used in the preceding section:

Lemma 7.5. The set of vectors

$$
e^{-k_{1} L} \pi\left(\sigma_{x}\left(I_{1}\right)\right) e^{-\left(k_{2}-k_{1}\right) L} \ldots e^{-\left(k_{n}-k_{n-1}\right) L} \pi\left(\sigma_{x}\left(I_{n}\right)\right) \Phi
$$

with $n \in \mathbb{N}, 0<k_{1}<k_{2}<\ldots<k_{n}$ and arbitrary finite subsets $I_{1}, \ldots, I_{n}$, is total.

Proof. Let $\Psi$ be orthogonal to all vectors of the form (7.8). Define

$$
F(z)=\left(\Psi, e^{i z_{1} L} \pi\left(\sigma_{x}\left(I_{1}\right)\right) e^{i z_{2} L} \ldots e^{i z_{n} L} \pi\left(\sigma_{x}\left(I_{n}\right)\right) \Phi\right) .
$$

By assumption, $F\left(i k_{1}^{\prime}, i k_{2}^{\prime}, \ldots i k_{n}^{\prime}\right)=0$ whenever $k_{1}^{\prime}, k_{2}^{\prime}, \ldots k_{n}^{\prime} \in \mathbb{N}$, where $k_{1}^{\prime}=k_{1}$, $k_{j}^{\prime}=k_{j}-k_{j-1}(j=2, \ldots, n)$. By exactly the same argument as before, we obtain $F(z)=0$. Hence

$$
\begin{array}{r}
\left(\Psi, \pi\left(\alpha_{z_{1}}\left(\sigma_{x}\left(I_{1}\right)\right)\right) \ldots \pi\left(\alpha_{z_{n}}\left(\sigma_{x}\left(I_{n}\right)\right)\right) \Phi\right) \\
\quad=F\left(z_{1}, z_{2}-z_{1}, \ldots, z_{n}-z_{n-1}\right)=0 .
\end{array}
$$

By Lemma 7.4, we obtain $(\Psi, \pi(A) \Phi)=0$ for all $A \in \mathfrak{A}$ and hence $\Psi=0$. Q.E.D.

\section{The $j$-Symmetry}

For conventional representation of Pauli spin matrices, the complex conjugation of matrix elements is a conjugate automorphism, leaving $\sigma_{x}$ and $\sigma_{z}$ invariant and changing $\sigma_{y}$ to $-\sigma_{y}$. Extending it to tensor products, we obtain an involutive 
conjugate automorphism $j$ of the (spin) $C^{*}$-algebra $\mathfrak{A}$, satisfying

$$
j\left(\sigma_{\mu}^{(k)}\right)=\varepsilon_{\mu} \sigma_{\mu}^{(k)}, \quad \varepsilon_{x}=\varepsilon_{z}=1, \quad \varepsilon_{y}=-1 .
$$

By (4.1) and (4.2), $j$ commutes with $\alpha_{i}^{\beta j M}(j=1,2)$, and hence

$$
j\left(F_{\beta}\right)=(\bar{F})_{\beta},
$$

where $\bar{F}$ is the complex conjugate of the function $F(\xi)$. The following proposition shows that $j$ is an analogue of the time reversal operator.

Proposition 8.1. Let a linear functional $\omega$ on the $C^{*}$-algebra generated by $\xi^{\prime} s$ be defined by

$$
\omega(F)=\varphi\left(F_{\beta}\right)
$$

with a ground state $\varphi$ of $\left(\mathfrak{U}, \alpha_{t}^{\beta}\right)$. Then $\omega$ is hermitian [i.e. $\omega(\bar{F})=\overline{\omega(\bar{F})}$ for all $\left.F\right]$ if and only if $\varphi$ is j-symmetric, i.e.

$$
\varphi(j(A))=\overline{\varphi(A)}, \quad A \in \mathfrak{U} .
$$

Proof. Let $\varphi$ satisfy (8.3). Then, for any $F$, we obtain

$$
\omega(\bar{F})=\varphi\left((\bar{F})_{\beta}\right)=\varphi\left(j\left(F_{\beta}\right)\right)=\overline{\varphi\left(F_{\beta}\right)}=\overline{\omega(F)}
$$

by (8.2) and (8.3). Hence $\omega$ is hermitian.

Conversely, let $\omega$ be hermitian. By the same computation

$$
\varphi\left(j\left(F_{\beta}\right)\right)=\varphi\left((\bar{F})_{\beta}\right)=\omega(\bar{F})=\overline{\omega(F)}=\overline{\varphi\left(F_{\beta}\right)} .
$$

By Lemma 7.4, $\left\{F_{\beta}\right\}$ is dense in $\mathfrak{A}$. Therefore, we obtain (8.3). Q.E.D. have

In the present case, $\psi_{\beta}$ is a state and hence $\varphi_{\beta}$ is $j$-symmetric. For $|\beta|>\beta_{c}$, we

$$
\left.\varphi_{\beta}(A)=\left(\varphi_{\beta+}(A)+\varphi_{\beta-}(A)\right) / 2=\overline{\varphi_{\beta}(j(A))}=\overline{\left(\varphi_{\beta+}(j(A))\right.}+\overline{\varphi_{\beta-}(j(A))}\right) / 2
$$

by the $j$-symmetry of $\varphi_{\beta}$. Since $\varphi_{\beta \pm}$ are mutually disjoint (in the sense of the associated representations) pure states, $\overline{\varphi_{\beta \pm}(j(A))}=\varphi_{\beta \pm}\left(j\left(A^{*}\right)\right), A \in \mathfrak{A}$, are also mutually disjoint pure states and we have the following alternatives for $\varphi_{ \pm} \equiv \varphi_{\beta \pm}$ :

$$
\begin{aligned}
& \text { (i) } \varphi_{ \pm}\left(j\left(A^{*}\right)\right)=\varphi_{ \pm}(A), \quad A \in \mathfrak{U} \text {. } \\
& \text { (ii) } \varphi_{ \pm}\left(j\left(A^{*}\right)\right)=\varphi_{\mp}(A), \quad A \in \mathfrak{U} \text {. }
\end{aligned}
$$

Namely both $\varphi_{\beta \pm}$ are $j$-symmetric or else $j$ interchanges them. The condition (ii) is equivalent to the $j \Theta$-symmetry of $\varphi_{ \pm}$due to $\varphi_{ \pm}(\Theta(A))=\varphi_{\mp}(A)$ :

$$
\text { (iii) } \varphi_{ \pm}\left(j \Theta\left(A^{*}\right)\right)=\varphi_{ \pm}(A), \quad A \in \mathfrak{U}
$$

The monomials

$$
A=\sigma_{x}\left(I_{x}\right) \sigma_{y}\left(I_{y}\right) \sigma_{z}\left(I_{z}\right)
$$

with mutually disjoint finite subsets $I_{x}, I_{y}, I_{z}$ of $\mathbb{Z}$ are total in $\mathfrak{A}$, where $\sigma_{\mu}(I)$ denotes $\prod_{j \in I} \sigma_{\mu}^{(j)}(\mu=x, y, z)$. Since $A^{*}=A$,

$$
j\left(A^{*}\right)=(-1)^{\left|I_{y}\right|} A, \quad j \Theta\left(A^{*}\right)=(-1)^{\left|I_{x}\right|} A,
$$


where $|I|$ denotes the cardinal of the set $I$. Thus

(i) holds if and only if $\varphi_{ \pm}(A)=0$ for $\left|I_{y}\right|$ odd,

(ii) holds if and only if $\varphi_{ \pm}(A)=0$ for $\left|I_{x}\right|$ odd.

Consider a domain of parameters in which $\varphi_{ \pm}(A)$ are (real) analytic in parameters for all $A$ [of the form (8.7)]. If $\varphi_{+}(A) \neq 0$ for one parameter value and for one $A_{1}$ with odd $\left|I_{x}\right|$, then $\varphi_{+}(A)$ is not identically 0 and (ii) is excluded [except possibly for zeros of $\varphi_{+}(A)$ when parameters vary]. Since (i) or (ii) holds, $\varphi_{ \pm}(A)=0$ for $\left|I_{y}\right|$ odd holds for all $A$ and all values of parameters in the domain, for which $\varphi_{+}\left(A_{1}\right) \neq 0$. By analyticity of $\varphi_{+}(A)$ (for $\left|I_{y}\right|$ odd), $\varphi_{ \pm}(A)=0$ holds for all $A$ with $\left|I_{y}\right|$ odd for all values of the parameters in the domain.

If this is not the case, the same argument proves that $\varphi_{ \pm}(A)=0$ for all $A$ with $\left|I_{x}\right|$ odd holds for all values of the parameters in the domain. Therefore, the choice between cases (i) and (ii) can be decided at one value of the parameters in the domain of analyticity.

For $K_{1}^{*}=0, \varphi_{ \pm}$are the pure ground state of the Hamiltonian $-K_{2} \sum_{j} \sigma_{x}^{(j)} \sigma_{x}^{(j+1)}$ and can be characterized by the property that it is annihilated by $1 \pm \sigma_{x}^{(j)}, j \in \mathbb{Z}$, where the same sign is taken for all $j$ if $K_{2}>0$ and an alternate sign is taken if $K_{2}<0$. Since $\sigma_{x}^{(j)}$ is $j$-invariant, the state so characterized has the $j$-symmetry. This proves the following.

Proposition 8.2. $\varphi_{\beta \pm}$ are j-symmetric.

The same argument leads to the following for the one-dimensional $X Y$-model [6].

Proposition 8.3. (1) if $|\lambda|<1$ and $\gamma>0$ in the one-dimensional $X Y$-model, then two pure ground states are j-symmetric and vanishes on $A$ of the form (8.7) with odd $\left|I_{y}\right|$.

(2) If $|\lambda|<1$ and $\gamma<0$, then two pure ground states are $j \Theta$-symmetric and vanishes on $A$ of the form (8.7) with odd $\left|I_{x}\right|$.

This shows occurrence of both cases (i) and (ii). The vanishing of states on specific $A$ 's of the above form has been given in an explicit evaluation in [14].

Proposition 8.2 can also be proved from the commutativity of $j$ with $\hat{\alpha}\left(U_{12}\right)$ of Sect. 3 via the automorphism method of [9]. (A comment due to D. E. Evans.) The alternatives (i) and (ii) can also be judged according to whether the following index takes the value 1 or -1 :

$$
\operatorname{ind}_{\left(C, \theta_{-}\right)} E=\operatorname{det}\left(\Gamma \theta_{-} C \mid P\right)(-1)^{[(\operatorname{dim} P) / 2]} .
$$

Here $C$ is the componentwise complex conjugation of $h \in l_{2}(\mathbb{Z}) \oplus l_{2}(\mathbb{Z})$ and induces $j$ on $\mathfrak{A}^{\mathrm{CAR}}$ by $j(B(h))=B(C h)(j(T)=T)$. The linear operator $\Gamma \theta_{-} C$ leaves the projection $P=E \wedge\left(1-\theta_{-} E \theta_{-}\right)$invariant and its restriction to the range of $P$ is a finite matrix $\Gamma \theta_{-} C \mid P$. The bracket denotes the integer not exceeding $(\operatorname{dim} P) / 2$. This index takes the value 1 or -1 due to $\left(\Gamma \theta_{-} C\right)^{2}=\mathbf{1}$, and can be shown to be invariant under any continuous deformation of $E$ (as long as $\Gamma E \Gamma=1-E$, $[E, C]=0$, and $E-\theta_{-} E \theta_{-}$is compact). Hence the alternatives (i) and (ii) can be judged at a particular value of parameters $\left(K_{1}^{*}, K_{2}\right)$ or $(\lambda, \gamma)$.

In this connection, we note a computational error in [5]: $U$ and $U^{*}$ in (6.8) of [5] are to be interchanged and, as a consequence, $f_{1}$ and $g_{1}$ are to be interchanged in (6.9), (6.10), and (6.11) of [5]. 
Acknowledgements. The author would like to thank Professors David E. Evans and Masuo Suzuki for their valuable comments.

\section{References}

1. Aizenman, M.: Transition invariance and instability of phase coexistence in the twodimensional Ising system. Commun. Math. Phys. 73, 83 (1980)

2. Araki, H.: Gibbs states of a one-dimensional quantum lattice. Commun. Math. Phys. 14, 120 (1969)

3. Araki, H: Publ. Res. Inst. Math. Sci. 6, 385 (1970)

4. Araki, H.: Ann. Sci. Éc. Norm. Super. 6, 67 (1973)

5. Araki, H., Evans, D.E.: On a $C^{*}$-algebra approach to phase transition in the two-dimensional Ising model. Commun. Math. Phys. 91, 489 (1983)

6. Araki, H., Matsui, T.: Commun. Math. Phys. 101, 213 (1985)

7. Araki, H., Matsui, T.: On analyticity of correlation functions for ground states of the $X Y$ model. Lett. Math. Phys. 11, 87 (1986)

8. Boas, R.P.: Entire functions. New York: Academic Press 1954

9. Evans, D.E., Lewis, J.T.: Commun. Math. Phys. 102, 521 (1986)

10. Gallavotti, G., Martin-Lof, A., Miracle-Solé, S.: In: Statistical mechanics and mathematical problems. Lenard, A. (ed.). Berlin, Heidelberg, New York: Springer 1973

11. Greenberg, W.: Critical temperature bounds of quantum lattice gases. Commun. Math. Phys. 13, 335 (1969)

12. Higuchi, Y.: In: Random fields. I. Fritz, J., Lebowitz, J.L., Szász, D. (eds.), p. 517. Colloquia Societatis Janos Bolyai, Vol. 27. Amsterdam, Oxford, New York: North-Holland 1981

13. Lanford III, O.E., Ruelle, D.: Observables at infinity and states with short range correlations in statistical mechanics. Commun. Math. Phys. 13, 194 (1969)

14. Matsui, T.: Explicit formulas for correlation functions of ground states of the 1-dimensional $X Y$-model, to appear in Ann. Inst. H. Poincaré

15. Suzuki, M.: Relationship among exactly soluble models of critical phenomena. I. Progr. Theor. Phys. 46, 1337 (1971)

Communicated by H. Araki

Received October 24, 1985; in revised form March 1, 1986 